\title{
The midgut transcriptome of Phlebotomus (Larroussius) perniciosus, a vector of Leishmania infantum: comparison of sugar fed and blood fed sand flies
}

Anna Dostálová ${ }^{1}$, Jan Votýpka', Amanda J Favreau², Kent D Barbian², Petr Volf ${ }^{1}$, Jesus G Valenzuela ${ }^{3^{*}}$ and Ryan C Jochim ${ }^{3 *}$

\begin{abstract}
Background: Parasite-vector interactions are fundamental in the transmission of vector-borne diseases such as leishmaniasis. Leishmania development in the vector sand fly is confined to the digestive tract, where sand fly midgut molecules interact with the parasites. In this work we sequenced and analyzed two midgut-specific cDNA libraries from sugar fed and blood fed female Phlebotomus perniciosus and compared the transcript expression profiles.

Results: A total of 4111 high quality sequences were obtained from the two libraries and assembled into 370 contigs and 1085 singletons. Molecules with putative roles in blood meal digestion, peritrophic matrix formation, immunity and response to oxidative stress were identified, including proteins that were not previously reported in sand flies. These molecules were evaluated relative to other published sand fly transcripts. Comparative analysis of the two libraries revealed transcripts differentially expressed in response to blood feeding. Molecules up regulated by blood feeding include a putative peritrophin (PperPer1), two chymotrypsin-like proteins (PperChym1 and PperChym2), a putative trypsin (PperTryp3) and four putative microvillar proteins (PperMVP1, 2, 4 and 5). Additionally, several transcripts were more abundant in the sugar fed midgut, such as two putative trypsins (PperTryp1 and PperTryp2), a chymotrypsin (PperChym3) and a microvillar protein (PperMVP3). We performed a detailed temporal expression profile analysis of the putative trypsin transcripts using qPCR and confirmed the expression of bloodinduced and blood-repressed trypsins. Trypsin expression was measured in Leishmania infantum-infected and uninfected sand flies, which identified the L. infantum-induced down regulation of PperTryp3 at 24 hours postblood meal.

Conclusion: This midgut tissue-specific transcriptome provides insight into the molecules expressed in the midgut of $P$. perniciosus, an important vector of visceral leishmaniasis in the Old World. Through the comparative analysis of the libraries we identified molecules differentially expressed during blood meal digestion. Additionally, this study provides a detailed comparison to transcripts of other sand flies. Moreover, our analysis of putative trypsins demonstrated that $L$. infantum infection can reduce the transcript abundance of trypsin PperTryp3 in the midgut of P. perniciosus.
\end{abstract}

\footnotetext{
* Correspondence: jvalenzuela@niaid.nih.gov; rjochim@niaid.nih.gov

${ }^{3}$ Vector Molecular Biology Unit, Laboratory of Malaria and Vector Research,

NIAID, NIH, Rockville, MD, 20852, USA

Full list of author information is available at the end of the article
} 


\section{Background}

Leishmaniases are a group of vector-borne diseases caused by parasitic protozoa of the genus Leishmania. Leishmania infantum (syn. L. chagasi) is the main etiological agent of zoonotic visceral leishmaniasis, the most deadly form of the disease. The lack of a human vaccine, increasing resistance to the currently used drugs and their serious side effects urge the need for research of visceral leishmaniasis. In the western and central part of the Mediterranean basin, the major vector of $L$. infantum is Phlebotomus perniciosus [1].

Leishmania amastigotes are ingested with the blood meal upon female sand fly feeding on the mammalian host. After a series of morphological changes, propagation and migration of the parasites to the anterior part of the midgut, the infection is transmitted to another host during the next blood feeding. In hematophagous arthropods, blood feeding induces a number of processes including digestion, metabolism, diuresis and egg development. Unlike many other arthropod-borne infections, e.g., Plasmodium in mosquitoes, Leishmania complete their whole developmental cycle within the midgut of the sand fly. Several natural barriers to Leishmania development in the midgut have been described including the secreted proteolytic enzymes, the peritrophic matrix surrounding the ingested blood meal and the necessity to bind to the midgut epithelium (reviewed by [2]). Thus, the midgut is the primary organ where interactions between the vector sand fly and the parasite occur and it represents a key target for interruption of Leishmania transmission.

While the genome sequences of several Leishmania species, including L. infantum, have been published [3] and molecular studies abound, molecular data on sand flies are limited. An analysis of expressed sequence tags (ESTs) from the whole Lutzomyia longipalpis sand fly and salivary gland transcriptomes of several sand fly species have been published (reviewed by [4]). With regard to Leishmania development in the midgut, particularly midgut-specific transcriptomic analyses, studies of $L$. longipalpis and Phlebotomus papatasi [5-7] have brought important insights into the repertoire of molecules expressed in the midgut. Several midgut proteins from these two species were functionally characterized [8] and shown to impact Leishmania development $[9,10]$.

In this study, we have generated and sequenced two cDNA libraries from the midgut tissue of $P$. perniciosus and analysed sequences present both before and after blood feeding. Furthermore, we provide phylogenetic analysis and comparison with the midgut molecules described in L. longipalpis and P. papatasi. Comparison of these three species is especially valuable with regard to Leishmania transmission. P. papatasi is the principal vector of cutaneous leishmaniasis caused by Leishmania major in the Old World [1]. It is refractory to the development of other species of Leishmania [11]. Lutzomyia longipalpis is the vector of L. infantum (chagasi) in Latin America and is considered a permissive vector due to full development of various Leishmania species in laboratory infections $[1,12]$. While being phylogenetically closer to $P$. papatasi, in some aspects $P$. perniciosus resembles $L$. longipalpis. First, it is a natural vector of $L$. infantum. Second, it is also permissive to the development of other Leishmania species [13]. Therefore, the present study provides a valuable database for identification of vector molecules that affect the vectorial competence of sand flies.

\section{Results and Discussion}

In order to gain insight into the spectrum of molecules present in the $P$. perniciosus midgut, two cDNA libraries from this organ were constructed, sequenced and analysed. The first library was constructed from a pool of midguts from sand flies allowed to feed on sucrose solution (sugar fed). For the construction of the second library (blood fed), midguts from sand flies 4-6 h, 24 h, 2, 3 and 4 days after blood feeding were pooled. These time points cover the course of blood digestion, allowing us to identify molecules transcribed in response to blood feeding. In total, 4511 clones were sequenced and 91\% of the sequences were of high quality and included in subsequent analyses. Analysis was performed on 2049 and 2062 sequences for the sugar fed and blood fed libraries, respectively. These sequences were deposited in the NCBI dbEST database under accession numbers [GenBank:GW815603-GW820028]. The comparable number of high quality sequences in each library allows for a better comparison of sequence abundance of specific molecules of interest in the libraries. The bioinformatic analyses of the sequences were performed using the dCAS cDNA annotation software [14]. Sequences were clustered together based on sequence homology and produced 207 and 163 contigs and 712 and 553 singletons (cluster with only one sequence) for the sugar fed and blood fed libraries, respectively. The average sequence per contig ratio was higher in the blood fed library (9.26) than in the sugar fed one (6.46), attributed to the strong induction of certain sequences after blood feeding (such as sequences coding for putative microvillar proteins, proteolytic enzymes and peritrophins, as discussed later). Combining the two libraries produced 370 contigs, 1085 singletons and an average ratio of 8.18 sequences per contig. Most of the clusters (890) had a significant $(\mathrm{E}<10 \mathrm{E}-5)$ BLASTX match to the NCBI nonredundant protein database. However, 565 clusters, mostly singletons, produced no match or low homology and these clusters likely represent transcript coding for novel proteins or potential non-coding regions. Clusters 
were assigned to general functional classes using the best match BLAST results of the KOG database as a guideline. The overall distribution of clusters in these functional classes in the two libraries is shown in Figure 1. The distribution illustrates the abundance of microvillar proteins and proteins involved in amino acid transport and metabolism (a category including proteolytic enzymes) after blood feeding.

The following paragraphs give a detailed description of the most abundant sequences identified in the libraries and sequences that are of interest with respect to the midgut physiology and Leishmania life cycle. The sequences, their putative functions and distribution in the two libraries are listed in Table 1 . Table 2 shows the best matches the sequences produced when compared to the NCBI non-redundant protein database using BLASTp.

\section{Trypsins}

Proteolytic enzymes were among the most abundant sequences detected in the libraries. Three putative trypsins were identified. PperTryp1 [GenBank:EZ933288], cluster 46, was one of the most abundant transcripts overall, strongly overrepresented in the sugar fed library (513 of 533 sequences). The putative protein has a predicted molecular weight of $27.6 \mathrm{kDa}$ after cleavage of the signal peptide and a pI of 5.41. PperTryp2 [GenBank:EZ933289], cluster 16, is a less abundant (10 sequences) putative trypsin that was only detected in the sugar fed library. The putative mature protein has a predicted molecular weight of $26.9 \mathrm{kDa}$ and a high pI of 8.83 (similar to a putative $P$. papatasi trypsin, PpTryp3 [GenBank:AAM96942]. Sequences coding for a third putative trypsin named PperTryp3 [GenBank:EZ933290]

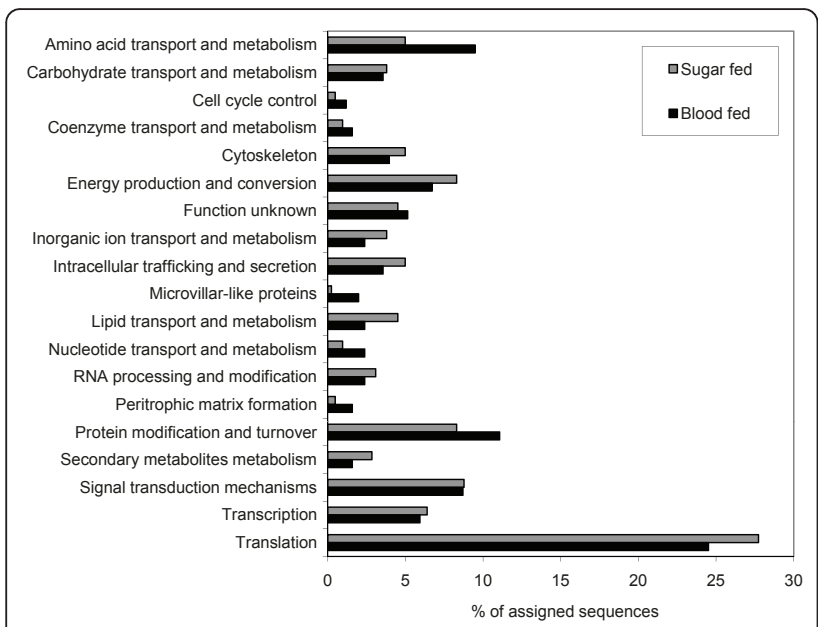

Figure 1 Distribution of clusters from the sugar fed and blood fed libraries in general functional classes. Significant match to the KOG database $(E<10 E-5)$ was used as a guideline for grouping the sequences into the functional classes. (cluster 63, 5' truncated) originated from the blood fed midgut library. In blood fed midguts we also identified a few partial transcripts, coding for a putative variant of this protein (5 sequences represented by clone PPRGFL_P8_H08, [GenBank:GW817404], Cluster 61). This Cluster 61 variant shows $82 \%$ identity to PperTryp3 at the amino acid level. Multiple sequence alignment of the putative $P$. perniciosus trypsin molecules (Figure 2) shows that structural cysteines, the H/D/S catalytic triad and putative substrate specifying residues are well conserved. Both PperTryp1 and PperTryp2, for which we obtained the full-length sequence of the transcripts, are pre-pro-peptides; having a predicted signal peptide and a putative pro-peptide cleavage site for activation of the mature protein.

In order to describe the expression dynamics of the identified putative trypsin molecules, we performed a qPCR analysis of the three transcripts before, and at several time points after, blood feeding. The results (Figure 3) correlate with the sequence abundance in the two libraries, proving the validity of the library comparison approach. In addition, the qPCR analysis provides a more detailed view of the trypsin expression after blood feeding. PperTryp1, the most abundant trypsin identified, was down regulated as soon as $6 \mathrm{~h}$ after blood feeding and further suppressed $24 \mathrm{~h}$ post-blood meal (about $1 / 50^{\text {th }}$ of pre-blood meal levels). Its expression returned to the pre-blood meal levels in the sand flies that had passed the remnants of blood meal. PperTryp 2 was detected in lower amounts (about $1 /$ $70^{\text {th }}$ of PperTryp 1 ) and represents another trypsin down regulated by blood feeding, with a time course similar to that observed for PperTryp1. In contrast, the qPCR analysis confirmed PperTryp3 as the main blood feedinginduced trypsin molecule. PperTryp 3 expression was already elevated after $6 \mathrm{~h}$ and the highest quantity of the transcripts was observed $24 \mathrm{~h}$ post-blood meal. PperTryp3 returned to negligible amounts in sand flies that had finished blood digestion.

This study brings the first expression analysis of sand fly trypsins using precise quantification by the means of qPCR. The observed $P$. perniciosus trypsin expression profile is in accordance with the results of earlier studies of $P$. papatasi and L. longipalpis midgut trypsin abundance from data acquired by semi-quantitative endpoint PCR $[15,16]$ and comparison of transcript abundance in cDNA libraries before and after blood feeding $[5,6]$. In all the three species, one or several trypsin transcripts (see Figure 4 for sequence accession numbers) are present in high abundance in sugar fed females while their quantities decrease after the intake of blood (PperTryp1, PperTryp2, LlTryp2, PpTryp1, PpTryp2). At the same time, the expression of other putative trypsins (PperTryp 3, LlTryp 1 and PpTryp4) is induced upon blood feeding. 
Table 1 Putative function and sequence abundance in the sugar fed (SF) and blood fed (BF) libraries

\begin{tabular}{|c|c|c|c|c|c|c|c|}
\hline Cluster & Clone & GenBank & Name & Putative function & SF & $\mathrm{BF}$ & Total \\
\hline 46 & PPRGUS_P2_F06 & EZ933288 & PperTryp1 & trypsin & 513 & 20 & 533 \\
\hline 16 & PPRGUM_P3_G08 & EZ933289 & PperTryp2 & trypsin & 10 & 0 & 10 \\
\hline 63 & PPRGFL_P8_E08 & EZ933290 & PperTryp3 & trypsin & 0 & 31 & 31 \\
\hline 81 & PPRGFL_P1_E01 & EZ933296 & PperChym1 & chymotrypsin & 0 & 82 & 82 \\
\hline 102 & PPRGFL_P7_D06 & EZ933297 & PperChym2 & chymotrypsin & 2 & 11 & 13 \\
\hline 1033 & PPRGUM_P1_A02 & EZ933298 & PperChym3 & chymotrypsin & 12 & 0 & 12 \\
\hline 816 & PPRGUM_P8_C08 & EZ933299 & PperChym4 & chymotrypsin & 7 & 0 & 7 \\
\hline 710 & PPRGUM_P7_F02 & EZ933300 & PperChym5 & chymotrypsin & 1 & 1 & 2 \\
\hline 539 & PPRGUL_P4_F08 & EZ966131 & PperCpepA & carboxypeptidase A & 2 & 1 & 3 \\
\hline 217 & PPRGFL_P5_D07 & EZ966132 & PperCpepB & carboxypeptidase B & 1 & 5 & 6 \\
\hline 126 & PPRGUL_P2_A02 & EZ966135 & PperApeptN & aminopeptidase $\mathrm{N}$ & 7 & 13 & 20 \\
\hline 84 & PPRGFM_P8_D04 & EZ966133 & PperAstacin1 & astacin & 4 & 8 & 12 \\
\hline 967 & PPRGUL_P6_A03 & EZ966134 & PperAstacin2 & astacin & 3 & 0 & 3 \\
\hline 45 & PPRGFL_P1_A01 & EZ933291 & PperMVP1 & microvillar protein & 0 & 681 & 681 \\
\hline 40 & PPRGFL_P3_G12 & EZ933292 & PperMVP2 & microvillar protein & 0 & 26 & 26 \\
\hline 18 & PPRGFL_P5_H01 & EZ933293 & PperMVP3 & microvillar protein & 18 & 6 & 24 \\
\hline 139 & PPRGFL_P5_G10 & EZ933294 & PperMVP4 & microvillar protein & 0 & 28 & 28 \\
\hline 52 & PPRGFL_P1_E11 & EZ933295 & PperMVP5 & microvillar protein & 0 & 35 & 35 \\
\hline 274 & PPRGFL_P7_G05 & EZ617708 & PperPGRPLB & peptidoglycan recognition protein LB & 1 & 1 & 2 \\
\hline 168 & PPRGFL_P5_C02 & EZ617707 & PperPGRPLC & peptidoglycan recognition protein $\mathrm{LC}$ & 0 & 1 & 1 \\
\hline 301 & PPRGFL_P8_G01 & EZ617706 & PperGNBP & gram-negative bacteria binding protein & 0 & 2 & 2 \\
\hline 163 & PPRGUM_P7_D04 & EZ617709 & PperGST1 & glutathione-S-transferase Sigma & 9 & 3 & 12 \\
\hline 463 & PPRGFM_P5_G03 & EZ617710 & PperGST2 & microsomal glutathione-S-transferase & 0 & 2 & 2 \\
\hline 1322 & PPRGUS_P3_F05 & EZ617711 & PperGST3 & glutathione-S-transferase Theta & 1 & 0 & 1 \\
\hline 729 & PPRGUM_P1_B05 & HM119220 & PperPRX & Peroxiredoxin & 1 & 0 & 1 \\
\hline 852 & PPRGUS-P1_C06 & EZ617712 & PperCat & Catalase & 9 & 0 & 9 \\
\hline 892 & PPRGUL_P4_B12 & EZ617713 & PperSOD1 & $\mathrm{Cu} / \mathrm{Zn}$ superoxide dismutase & 1 & 0 & 1 \\
\hline 1166 & PPRGUM_P4_E03 & EZ617714 & PperSOD2 & $\mathrm{Cu} / \mathrm{Zn}$ superoxide dismutase & 1 & 0 & 1 \\
\hline 373 & PPRGFM_P2_E03 & EZ617715 & PperXDH & xanthine dehydrogenase & 1 & 1 & 2 \\
\hline 88 & PPRGFM_P2_G07 & EZ617716 & PperFLC & ferritin light chain & 5 & 6 & 11 \\
\hline 332 & PPRGUS-P1_A12 & EZ617717 & PperFHC & ferritin heavy chain & 9 & 7 & 16 \\
\hline 330 & PPRGFS_P8_D12 & HM119221 & PperPer2 & peritrophin & 1 & 4 & 5 \\
\hline 156 & PPRGUM_P7_A09 & HM119222 & PperPer3 & peritrophin & 1 & 2 & 3 \\
\hline 97 & PPRGFL_P6_E01 & EZ933302 & PperPer1 & peritrophin & 0 & 94 & 94 \\
\hline 124 & PPRGFL_P2_E05 & EZ933285 & PperChit & chitinase & 0 & 3 & 3 \\
\hline 358 & PPRGUM_P6_A03 & EZ933286 & cluster 358 & PM formation/unknown & 10 & 3 & 13 \\
\hline 379 & PPRGFM_P6_A06 & EZ933287 & cluster 379 & PM formation/unknown & 2 & 4 & 6 \\
\hline 174 & PPRGFL_P4_A04 & HQ015444 & PperGH13 & glycoside hydrolyse & 23 & 9 & 32 \\
\hline 183 & PPRGFM_P6_G03 & HQ015443 & PperGH31 & glycoside hydrolyse & 12 & 3 & 15 \\
\hline 79 & PPRGFL_P6_G10 & HQ015441 & cluster 79 & lipid recognition/unknown & 0 & 9 & 9 \\
\hline 652 & PPRGUS_P7_D02 & HQ015442 & PperSA & $40 S$ ribosomal protein SA & 8 & 1 & 9 \\
\hline 461 & PPRGUM_P6_G08 & EZ933301 & PperS7 & ribosomal protein S7 & 6 & 2 & 8 \\
\hline
\end{tabular}

Phylogenetic analysis of the putative trypsins (Figure 4) shows that the sequences abundant before blood feeding share similarity and, together with LuloTryp4 (reported in similar numbers both from blood fed and sugar fed L. longipalpis sand flies), form a clade apart from the other sand fly trypsins that include PperTryp3. The predicted pre-pro-peptide structure and high abundance of transcripts in sugar fed sand flies together with the virtual absence of trypsin-like enzymatic activity in sugar fed sand fly midguts [17] suggests that PperTryp1, PperTryp2 and their respective homologs are associated with initial blood meal digestion as they can be quickly translated and processed following blood feeding.

The onslaught of proteolytic activity after the intake of blood is one of the barriers for Leishmania development in the midgut [2]. Sant'Anna et al. [10] have shown that 
Table 2 Selected clusters of combined $P$. perniciosus midgut CDNA libraries: best match to the NCBI non-redundant protein database

\begin{tabular}{|c|c|c|c|c|c|}
\hline Cluster & GenBank & Name & Best match to $\mathrm{nr}$ protein database & Best match GenBank & NR E value \\
\hline 46 & EZ933288 & PperTryp1 & trypsin 2 [Lutzomyia longipalpis] & ABM26905.1 & $3.00 \mathrm{E}-82$ \\
\hline 16 & EZ933289 & PperTryp2 & trypsin 1 [Phlebotomus papatasi] & AAM96940.1 & $3.00 \mathrm{E}-76$ \\
\hline 63 & EZ933290 & PperTryp3 & putative trypsin 3 [Lutzomyia longipalpis] & ABV60308.1 & $1.00 \mathrm{E}-92$ \\
\hline 81 & EZ933296 & PperChym1 & putative chymotrypsin [Lutzomyia longipalpis] & ABV60294.1 & 1.00E-109 \\
\hline 102 & EZ933297 & PperChym2 & chymotrypsin-like protein [Phlebotomus papatasi] & ABV44728.1 & 4.00E-94 \\
\hline 1033 & EZ933298 & PperChym3 & putative chymotrypsin [Lutzomyia longipalpis] & ABV60294.1 & $8.00 \mathrm{E}-77$ \\
\hline 816 & EZ933299 & PperChym4 & putative chymotrypsin [Lutzomyia longipalpis] & ABV60293.1 & $6.00 \mathrm{E}-58$ \\
\hline 710 & EZ933300 & PperChym5 & serine protease1/2 [Culex quinquefasciatus] & XP_001845462.1 & $1.00 \mathrm{E}-59$ \\
\hline 539 & EZ966131 & PperCpepA & carboxypeptidase A [Aedes aegypti] & ААТ36730.1 & 1.00E-116 \\
\hline 217 & EZ966132 & PperCpepB & carboxypeptidase B-like protein [Phlebotomus papatasi] & ABV44754.1 & $1.00 \mathrm{E}-170$ \\
\hline 126 & EZ966135 & PperApeptN & aminopeptidase N [Aedes aegypti] & AAK73351.1 & $1.00 \mathrm{E}-44$ \\
\hline 84 & EZ966133 & PperAstacin 1 & astacin-like metalloprotease [Lutzomyia longipalpis] & ABV60299.1 & 1.00E-92 \\
\hline 967 & EZ966134 & PperAstacin2 & AGAP010758-PA [Anopheles gambiae] & XP_318553.4 & $2.00 \mathrm{E}-49$ \\
\hline 45 & EZ933291 & PperMVP1 & microvillar-like protein 1 [Lutzomyia longipalpis] & ABV60289.1 & $2.00 E-69$ \\
\hline 40 & EZ933292 & PperMVP2 & microvilli-like protein 2 [Phlebotomus papatasi] & ABV44759.1 & $7.00 \mathrm{E}-69$ \\
\hline 18 & EZ933293 & PperMVP3 & microvilli-like protein 3 [Phlebotomus papatasi] & ABV44760.1 & $6.00 \mathrm{E}-57$ \\
\hline 139 & EZ933294 & PperMVP4 & microvilli-like protein [Phlebotomus papatasi] & ABV44761.1 & $2.00 \mathrm{E}-92$ \\
\hline 52 & EZ933295 & PperMVP5 & microvillar-like protein [Lutzomyia longipalpis] & ABV60295.1 & $1.00 \mathrm{E}-67$ \\
\hline 274 & EZ617708 & PperPGRPLB & putative PGRP [Phlebotomus papatasi] & ABV60369.1 & 1.00E-104 \\
\hline 168 & EZ617707 & PperPGRPLC & PGRP-IC isoform [Anopheles gambiae] & AGAP005203-PC & 4.00E-62 \\
\hline 301 & EZ617706 & PperGNBP & GNBP [Aedes aegypti] & XP_001664288 & $1.00 \mathrm{E}-77$ \\
\hline 163 & EZ617709 & PperGST1 & GST-like protein [Phlebotomus papatasi] & ABV44736.1 & $3.00 \mathrm{E}-113$ \\
\hline 463 & EZ617710 & PperGST2 & microsomal GST [Culex quinquefasciatus] & XP_001863047.1 & $8.00 \mathrm{E}-20$ \\
\hline 1322 & EZ617711 & PperGST3 & GST theta [Aedes aegypti] & XP_001659667.1 & $8.00 \mathrm{E}-22$ \\
\hline 729 & HM119220 & PperPRX & peroxiredoxin-like [Phlebotomus papatasi] & ABV44727.1 & $6.00 \mathrm{E}-86$ \\
\hline 852 & EZ617712 & PperCat & putative catalase [Lutzomyia longipalpis] & ABV60342.1 & $0.00 \mathrm{E}+00$ \\
\hline 892 & EZ617713 & PperSOD1 & putative Cu/Zn SOD [Lutzomyia longipalpis] & ABV60343.1 & $5.00 \mathrm{E}-89$ \\
\hline 1166 & EZ617714 & PperSOD2 & superoxide dismutase [Culex quinquefasciatus] & XP_001866335 & $9.00 \mathrm{E}-64$ \\
\hline 373 & EZ617715 & PperXDH & XDH [Lutzomyia longipalpis] & CAP08999.1 & $4.00 \mathrm{E}-45$ \\
\hline 88 & EZ617716 & PperFLC & FLC-like [Phlebotomus papatasi] & ABV44741.1 & 1.00E-111 \\
\hline 332 & EZ617717 & PperFHC & FHC-like [Phlebotomus papatasi] & ABV44737 & $7.00 \mathrm{E}-73$ \\
\hline 330 & HM119221 & PperPer2 & putative peritrophin [Lutzomyia longipalpis] & ABV60320.1 & 2.00E-09 \\
\hline 156 & HM119222 & PperPer3 & peritrophin-like protein [Phlebotomus papatasi] & ABV44751.1 & $4.00 \mathrm{E}-59$ \\
\hline 97 & EZ933302 & PperPer1 & peritrophin-like protein [Phlebotomus papatasi] & ABV44705.1 & 1.00E-102 \\
\hline 124 & EZ933285 & PperChit & midgut chitinase [Phlebotomus papatasi] & AAV49322.1 & 4.00E-61 \\
\hline 358 & EZ933286 & cluster 358 & 14.5 kDa salivary protein [Phlebotomus duboscqi] & ABI20163 & $3.00 E-49$ \\
\hline 379 & EZ933287 & cluster 379 & $31.5 \mathrm{kDa}$ midgut protein [Phlebotomus papatasi] & ABV44721.1 & $5.00 \mathrm{E}-90$ \\
\hline 174 & HQ015444 & PperGH13 & alpha-amylase [Aedes aegypti] & XP_001649787.1 & 1.00E-170 \\
\hline 183 & HQ015443 & PperGH31 & GK14321 [Drosophila willistoni] & XP_002073831.1 & $0.00 \mathrm{E}+00$ \\
\hline 79 & HQ015441 & cluster 79 & Niemann-Pick Type C2, putative [Aedes aegypti] & XP_001647805.1 & $2.00 \mathrm{E}-14$ \\
\hline 652 & HQ015442 & PperSA & 40 S ribosomal protein SA [Simulium nigrimanum] & ACZ28384.1 & $1.00 \mathrm{E}-68$ \\
\hline 461 & EZ933301 & PperS7 & $40 \mathrm{~S}$ ribosomal protein S7-like protein [Phlebotomus papatasi] & ABV44745.1 & 2.00E-95 \\
\hline
\end{tabular}

suppression of the major blood meal-induced trypsin (LlTryp1) in L. longipalpis by the means of RNAi enhances the survival of L. mexicana in the midgut. Some studies have demonstrated the ability of L. major and L. infantum to suppress or delay the peak of trypsin activity in the midgut [18-20]. Transcriptomic studies $[5,6]$ have also shown modulation of trypsin-like transcript abundance in P. papatasi and L. longipalpis midgut in the presence of Leishmania parasites. qPCR was used to measure trypsin expression in infected sand flies to evaluate modulation of the $P$. perniciosus midgut trypsins by $L$. infantum. Our analysis showed that the amount of PperTryp3, the major blood meal-induced trypsin, is decreased in the presence of L. infantum 

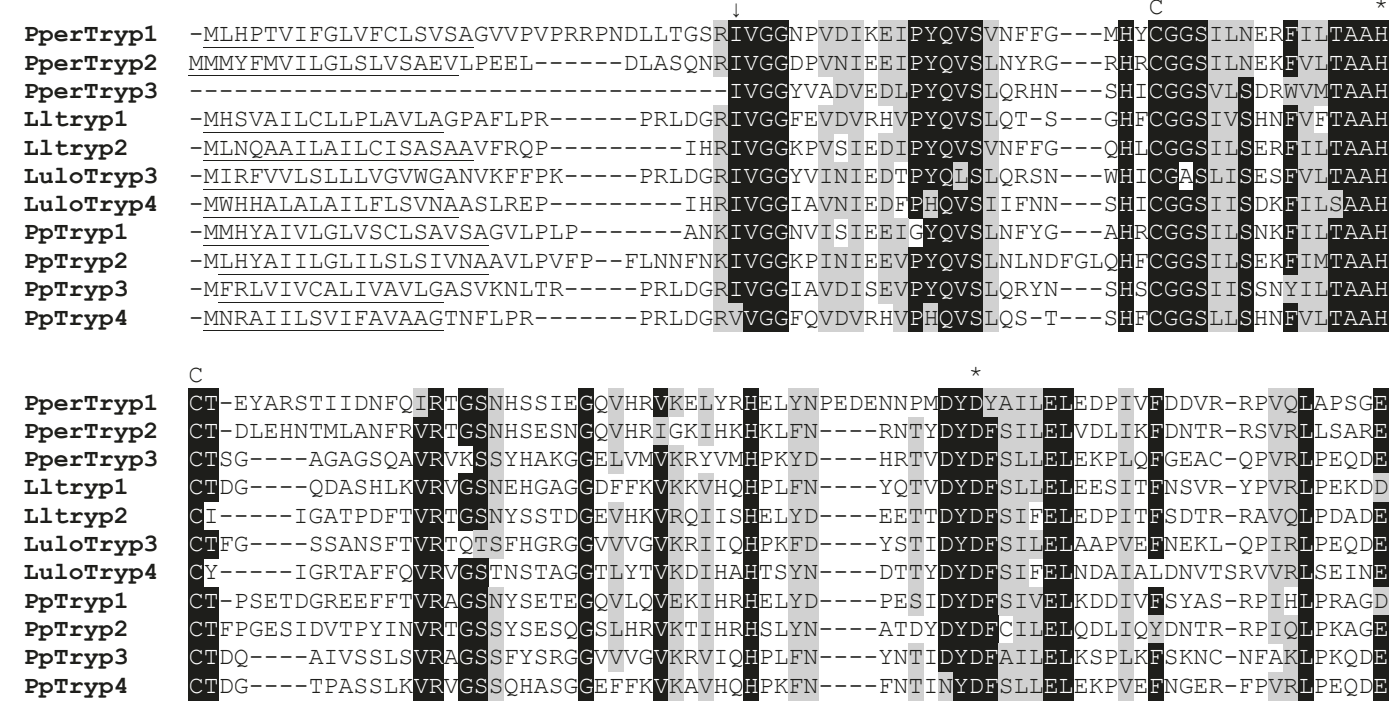

PperTryp1
PperTryp2
PperTryp3
Lltryp1
Lltryp2
LuloTryp3
LuloTryp4
PpTryp1
PpTryp2
PpTryp3
PpTryp4
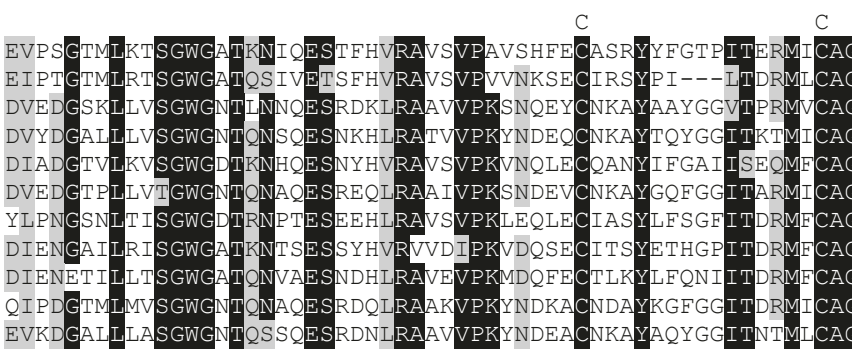

$\#$ C *
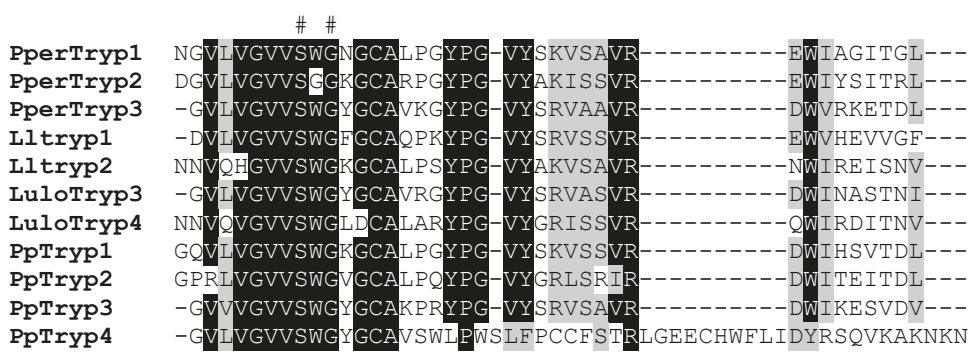

Figure 2 Multiple sequence alignment of putative sand fly trypsins. Pper: Phlebotomus perniciosus, Pp: Phlebotomus papatasi, Lulo: Lutzomyia longipalpis. Predicted signal peptides are underlined, the putative activation cleavage site is indicated by ( $\downarrow$ ), conserved cysteines (C), catalytic H/D/S residues marked by (*) and substrate binding site marked by (\#). Accession numbers: PperTryp1 [GenBank:EZ933288], PperTryp2 [GenBank:EZ933289], PperTryp3 [GenBank:EZ933290], Lltryp1 [GenBank:ABM26904], Lltryp2 [GenBank:ABM26905], LuloTryp3 [GenBank:ABV60308], LuloTryp4 [GenBank: ABV60300], PpTryp1 [GenBank:AAM96940], PpTryp2 [GenBank:AAM96941], PpTryp3 [GenBank:AAM96942], PpTryp4 [GenBank: AAM96943].

(Figure 3). This difference was observed in sand flies 24 hours post-blood meal, which correlated with the peak of PperTryp3 expression in uninfected blood fed sand flies. Our findings suggest the ability of L. infantum to suppress or delay the expression of the major bloodinduced trypsin in P. perniciosus and identify this molecule as an interesting candidate for future studies.

\section{Chymotrypsins}

Chymotrypsin-like enzymes are another group of proteases found in abundance in the midgut of sand flies and mosquitoes. Five clusters coding for putative chymotrypsins were identified in the libraries and each cluster was 5' truncated. PperChym1 [GenBank:EZ933296], cluster 81 , was the most abundant and was only detected in the blood fed midgut library (82 sequences). Similarly, the second most abundant chymotrypsin-like sequence, PperChym2 [GenBank:EZ933297], cluster 102, probably codes for a digestive enzyme up-regulated by blood feeding as 11 of the 13 sequences were found in the blood fed library. In contrast, PperChym3 [GenBank: EZ933298], cluster 1033, and PperChym4 [GenBank: 


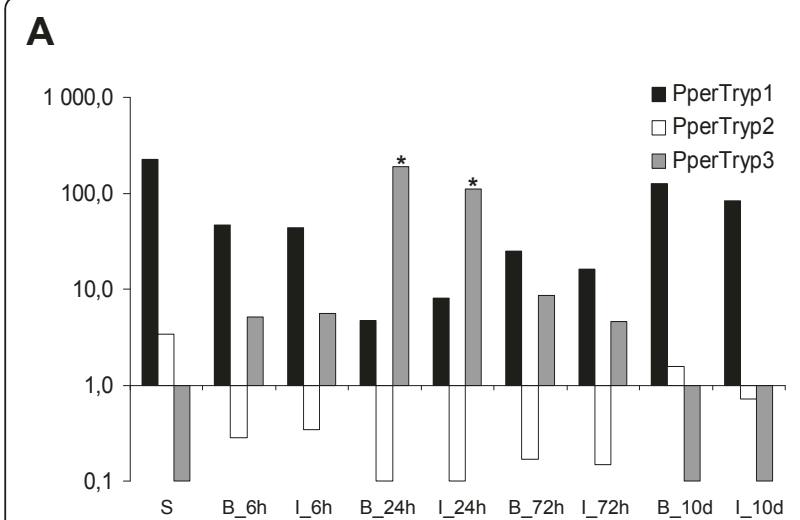

B

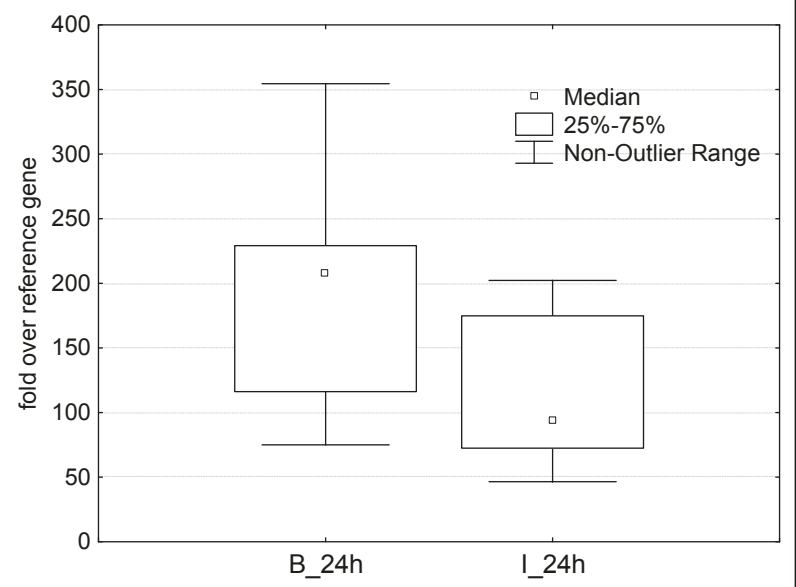

Figure 3 Influence of blood feeding and $L$. infantum infection on the dynamics of $P$. perniciosus trypsins expression. (A) The graph shows PperTryp1, PperTryp2 and PperTryp3 expression as fold over the reference housekeeping gene (PpPerS7 ribosomal protein) before and after the blood feeding ( 6 hours, 24 hours, 72 hours and 10 days). Each column represents the mean of ten females. S, sugar fed sand flies; B, blood fed sand flies; I, blood fed and L. infantum infected sand flies. The statistically significant difference between the infected and uninfected sand flies is indicated by $\left(^{*}\right)$. (B) The graph shows significant difference of PperTryp3 expression in uninfected (B_24) and infected (I_24) sand flies 24 hours after blood feeding; Mann-Whitney $U$ Test $(U=20 ; Z=2.268), p=0.023$.

EZ933299], cluster 816, sequences were only found in the sugar fed sand fly library. The expression pattern of these chymotrypsins is similar to the aforementioned trypsin molecules indicating that there may be early and late classes of serine proteases in sand flies, similar to what has been observed in mosquitoes[21] The least abundant putative chymotrypsin sequence PperChym5 [GenBank: EZ933300], cluster 710, was represented by one sequence in each of the libraries. The phylogenetic analysis of amino acid sequences (Figure 5) shows conservation in sequence homology of PperChym1-4 with putative P. papatasi and L. longipalpis midgut chymotrypsins. PperChym2 formed

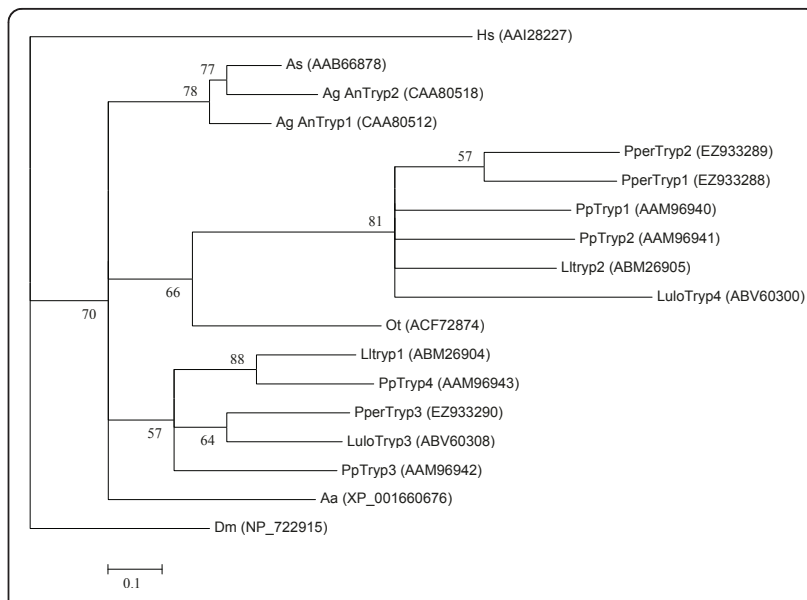

Figure 4 Phylogenetic analysis of putative trypsins from Anopheles stephensi (As), Ochlerotatus taeniorhynchus (Ot), Homo sapiens (Hs), Aedes aegypti (Aa), Anopheles gambiae (Ag), Phlebotomus perniciosus (Pper), Lutzomyia longipalpis (Lulo), Phlebotomus papatasi $(\mathrm{Pp})$ and Drosophila melanogaster (Dm) GenBank accession numbers are given in parentheses and node support is indicated by the bootstrap values.

a subclade along with LuloChym2 and Ppchym3. PperChym5 is more distantly related to putative chymotrypsins described in other sand flies. The most similar sequence in the NCBI non-redundant protein database is a putative serine protease of Culex quinquefasciatus [GenBank:XP_001845462.1] $(\mathrm{E}=1 \mathrm{e}-59)$ and the best match in the Swissprot database is the white shrimp, Litopenaeus vannamei, Chymotrypsin BI [Swiss-Prot: Q00871, E = 3 e36 with a proven chymotrypsin catalytic activity. Also, a conserved serine residue at the substrate specifying site suggests a chymotrypsin-like specificity of PperChym 5 enzyme (Figure 6). The H/D/S catalytic triad and cysteine residues are well conserved among all the putative $P$. perniciosus chymotrypsin sequences. Putative chymotrypsin transcript abundance has previously been shown to be altered by Leishmania infection in the midgut. LuloChym1A in L. longipalpis and PpChym2 in P. papatasi were reported as underrepresented in the midgut in the presence of $L$. infantum and $L$. major, respectively $[5,6]$.

\section{Carboxypeptidases}

A number of clusters coding for putative proteins with homology to carboxypeptidases were identified. Two putative metallo-carboxypeptidases of the M14 A/B subfamily were named PperCpepA and PperCpepB. PperCpepA [GenBank:EZ966131] (cluster 539, 5' truncated) shows similarity to carboxypeptidases A described from mosquitoes. Phylogenetically, PperCpepA clusters more distantly from the carboxypeptidases A described in the midgut of other phlebotomine species (Figure 7). Carboxypeptidase A, a zinc-metalloprotease, can hydrolyze 


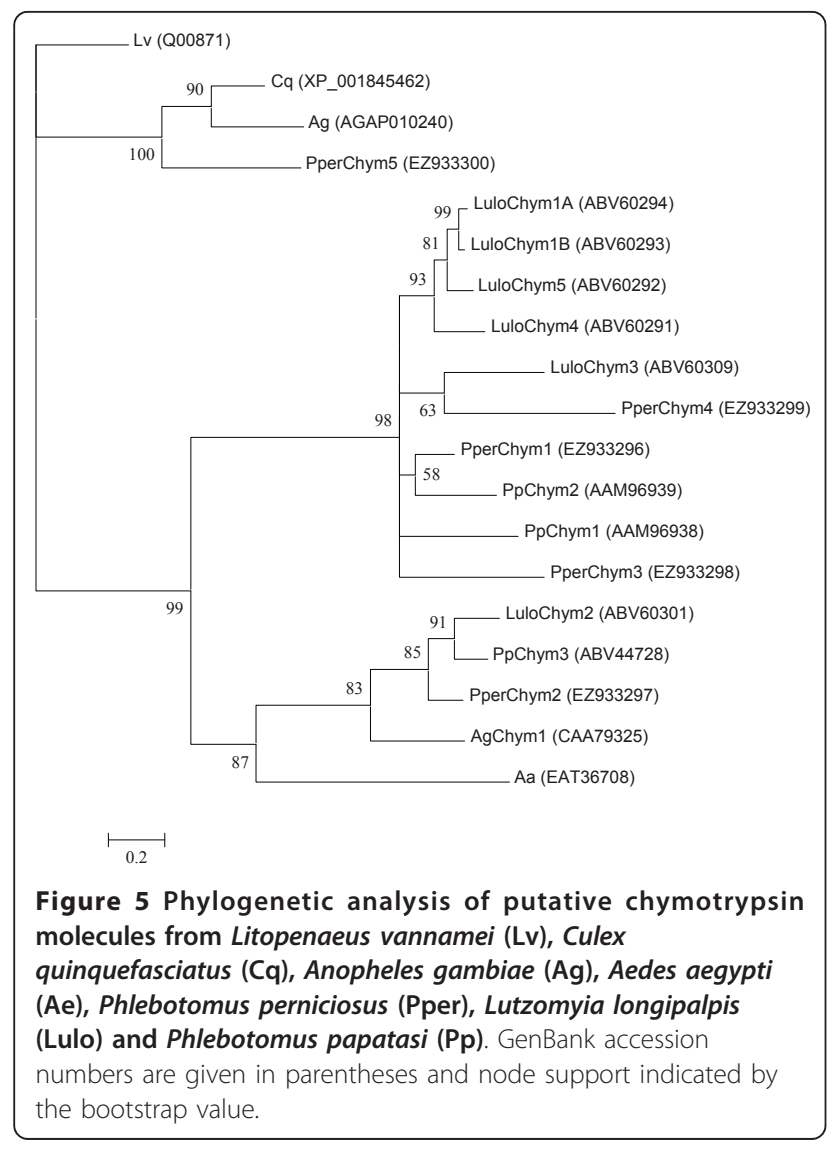

aromatic and aliphatic side chains from the $\mathrm{C}$-terminus. PperCpepB [GenBank:EZ966132] (cluster 217, 5' truncated) is similar to mosquito and sand fly midgut carboxypeptidase B. Carboxypeptidase B specifically hydrolyzes C-terminal arginine and lysine. PperCpepB possesses the conserved aspartate residue at the position responsible for this specific substrate recognition [22] (Figure 8 ). Due to the low number of sequences in this cluster a comparative analysis between the sugar fed and blood fed libraries was not possible; however, it is notable that five of the six sequences of PperCpepB were contributed by the blood fed library. Anopheles gambiae midgut carboxypeptidase B has been shown to be up-regulated by Plasmodium infection and antibodies against one of these enzymes, CPBAg1 [GenBank: CAF28572] blocked parasite development in the mosquito midgut [23]. In L. longipalpis, one of the carboxypeptidases transcripts, LuloCpepA1, [GenBank: ABV60310] was underrepresented in a cDNA library from $L$. infantum-infected midgut as compared to uninfected sand flies [6].

\section{Aminopeptidases}

A partial transcript coding for a putative alanyl aminopeptidase was identified (cluster 126). The molecule, named PperApeptN [GenBank:EZ966135], is similar to mosquito membrane aminopeptidases of the M1 family (aminopeptidase N). It was abundant in both libraries (13 and 7 sequences in blood fed and sugar fed midgut libraries, respectively). Membrane alanyl aminopeptidases were described in the midgut of many Dipteran species including mosquitoes, where they were identified as receptors for Plasmodium ookinetes and also Bacillus thuringiensis Cry toxin binding [24,25]. In the sand fly midgut, aminopeptidase activity was detected after blood feeding, mainly associated with the midgut wall (using leucine-p-nitroanilide LpNA as a substrate) [17]. This activity was reduced in $P$. papatasi and $P$. langeroni following infection with L. major [19].

\section{Astacins}

Two clusters coding for putative astacin-like zinc metalloproteases were identified in the libraries. The more abundant cluster, PperAstacin1 [GenBank:EZ966133], cluster 84 , is predicted to encode a protein with a molecular weight of $27.0 \mathrm{kDa}$ once secreted and $\mathrm{pI}$ of 5.05 . It was present both in the sugar fed and blood fed libraries. The transcript of cluster 967 was named PperAstacin2 [GenBank:EZ966134] and the predicted translated product has a molecular weight of 26.5 and pI 6.00 after cleavage of the signal peptide. It was only detected in the sugar fed library. Phylogenetic analysis of other putative astacin sequences shows that PperAstacin 1 is similar to astacin-like molecules previously described in L. longipalpis, LuloAstacin, [GenBank: ABV60299] P. papatasi and other Diptera. PperAstacin2 is most similar to a putative astacin from A. gambiae using BLASTp similarity search of the NCBI non-redundant protein database. However, in a phylogenetic analysis it branches away from all other Dipteran sequences (Figure 9A). Multiple sequence alignment (Figure 9B) shows the differences in amino acid sequences and illustrates the conservation of all residues likely responsible for zinc-binding and catalytic activity in the putative $P$. perniciosus astacins.

\section{Microvillar proteins}

The most abundant transcripts identified in the library were sequences coding for proteins with similarity to major insect allergen proteins. These insect-specific proteins containing insect-allergen domains [InterPro: IPR010629] were first described as the major human allergens in the faeces of the cockroaches Blatella germanica and Periplaneta americana [26]. In butterflies of the Pieridae family, a novel family of proteins with multiple insect-allergen domains has evolved (nitrile-specifier protein family) to serve a role in detoxification of plant metabolites in the butterfly larvae food $[27,28]$. In mosquitoes, proteins with a single insect-allergen 


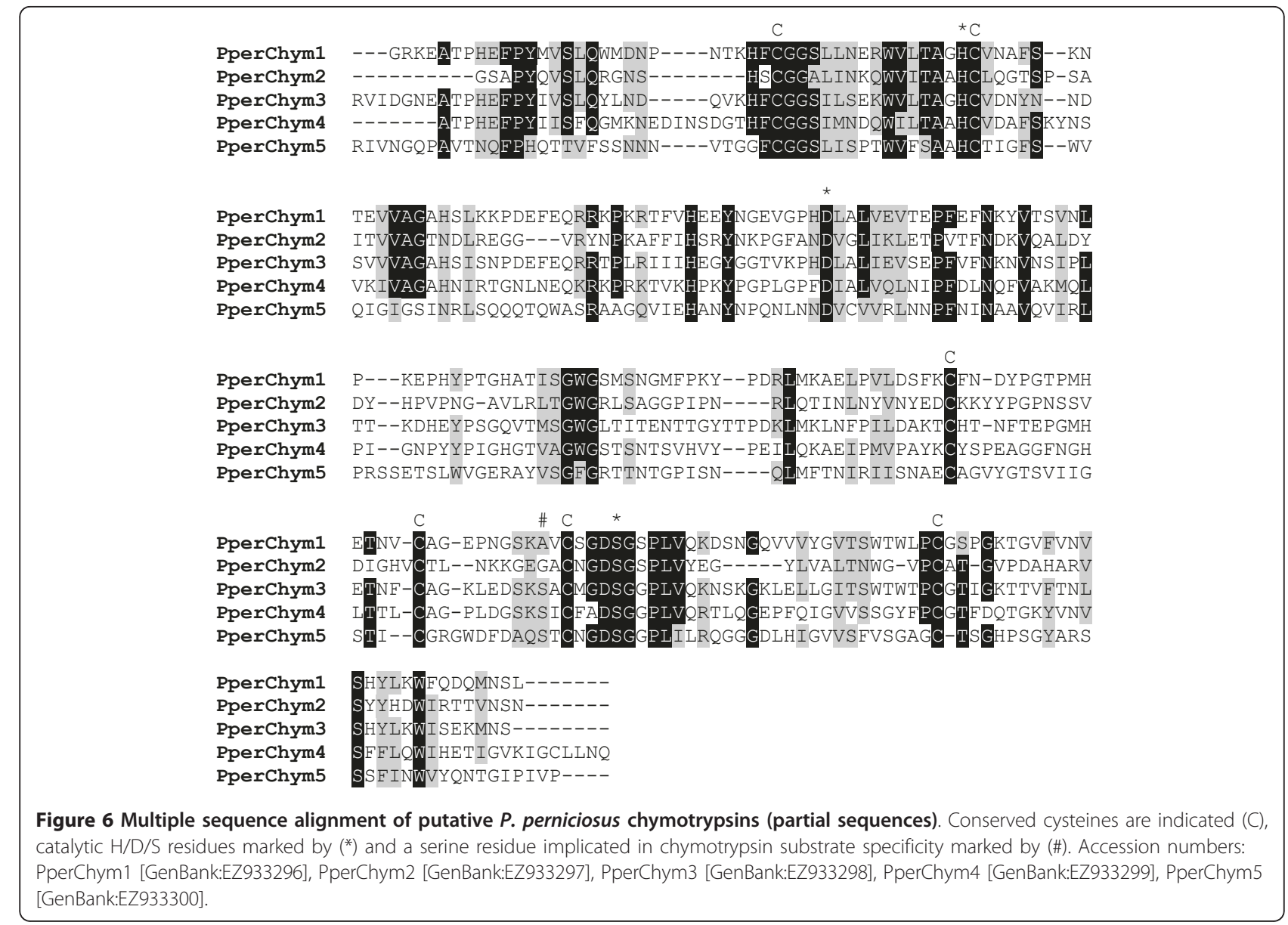

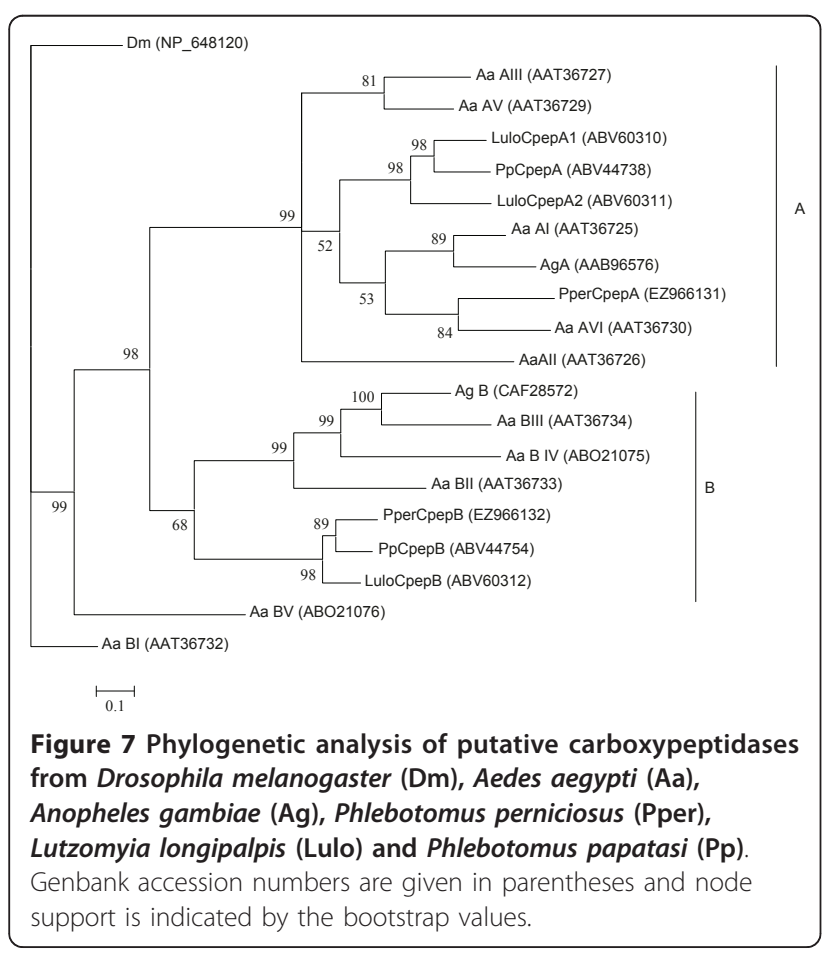

domain have been identified and termed G12 microvillar proteins. These molecules have been shown to be induced in the mosquito midgut after blood-feeding $[29,30]$. In Aedes aegypti, the G12 protein, AEG12, [GenBank:AAL05408.1] has been shown to be expressed only in the midgut after blood feeding and located on the microvillar membranes of the midgut epithelial cells [30]. The role of insect allergen proteins, other than nitrile-specifier protein family in Pieridae, has not yet been characterized.

We identified five putative homologs of the insectallergen proteins in the $P$. perniciosus libraries. These putative microvillar proteins (MVPs) possess a predicted signal peptide (where full-length sequences were obtained) and a single insect-allergen domain. PperMVP1 [GenBank:EZ933291] encodes a putative protein with a mature molecular weight of $21.4 \mathrm{kDa}$ and $\mathrm{pI}$ of 5.16. Derived from 681 sequences (cluster 45 and variants) PperMVP1 was the most abundant transcript overall. Transcripts of PperMVP1, and three other $P$. perniciosus MVPs, were only found in the blood fed cDNA library. These other blood feeding induced MVPs include PperMVP2 [GenBank:EZ933292] (cluster 40 and 


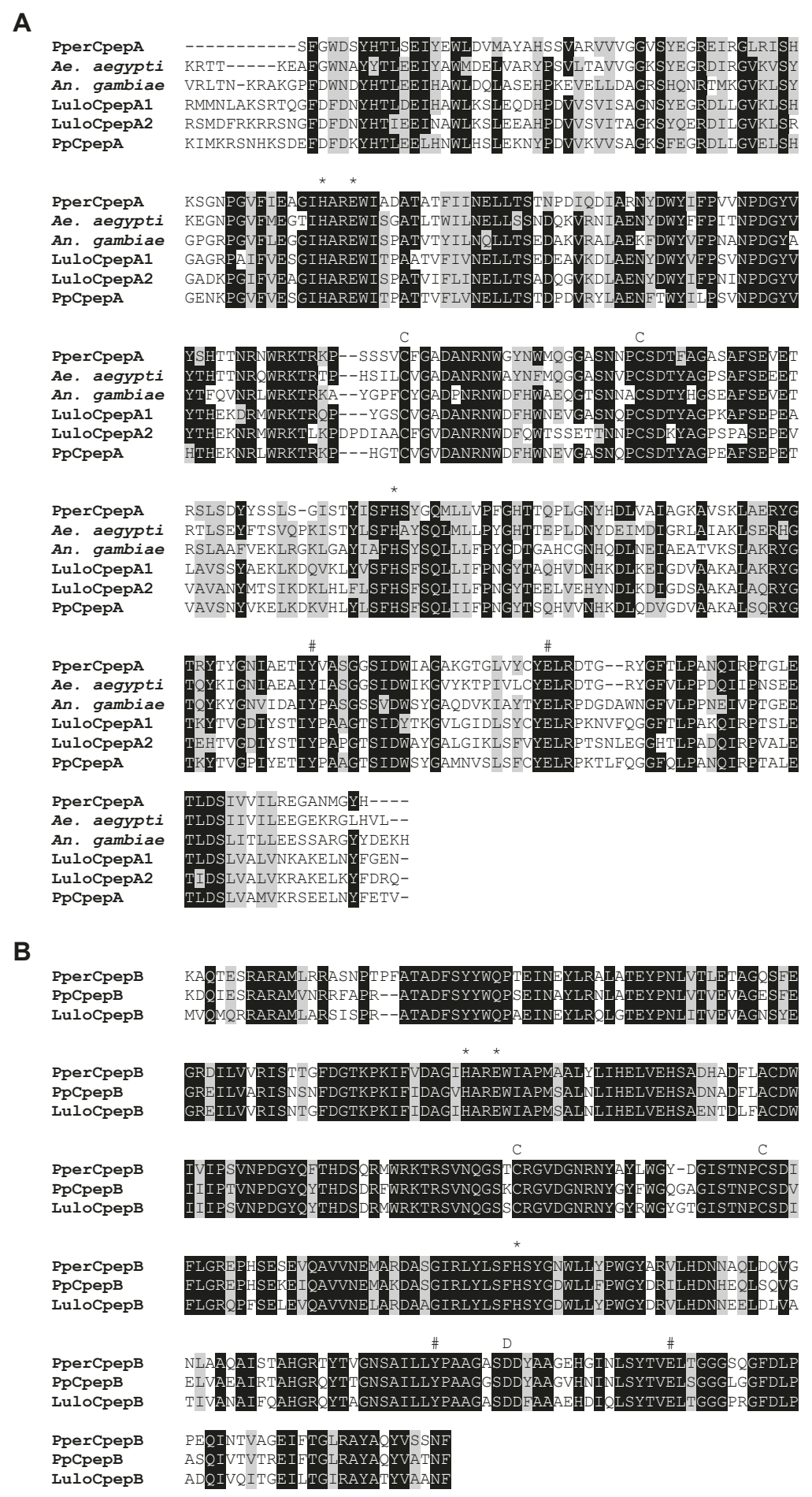

Figure 8 Sequence alignment of putative midgut carboxypeptidases. (A) Comparison of mature Carboxypeptidase A proteins of Aedes aegypti (A. aegypti), Anopheles gambiae (A. gambiae), Phlebotomus perniciosus (Pper), Phlebotomus papatasi (Pp) and Lutzomyia longipalpis (Lulo). $\mathrm{N}$-terminal portion of the peptides are not shown due to PperCpepA $5^{\prime}$ mRNA truncation. Conserved cysteines are indicated (C), metal binding residues are marked by $\left(^{*}\right)$ and catalytic residues are marked by (\#). (B) Comparison of mature Carboxypeptidase B proteins of $P$. perniciosus (Pper), P. papatasi (Pp) and L. longipalpis (Lulo). Conserved cysteines are indicated (C), metal binding residues are marked by $\left(^{*}\right)$, catalytic residues are marked by (\#) and a conserved aspartate in the binding pocket of carboxypeptidases B is indicated (D). Accession numbers: PperCpepA [GenBank:EZ966131], A. aegypti [GenBank:AAT36730], A. gambiae [GenBank:AAB96576], LuloCpepA1 [GenBank:ABV60310], LuloCpepA2 [GenBank: ABV60311], PpCpepA [GenBank:ABV44738], PperCpepB [GenBank:EZ966132], PpCpepB [GenBank:ABV44754], LuloCpepB [GenBank:ABV60312]. 
A

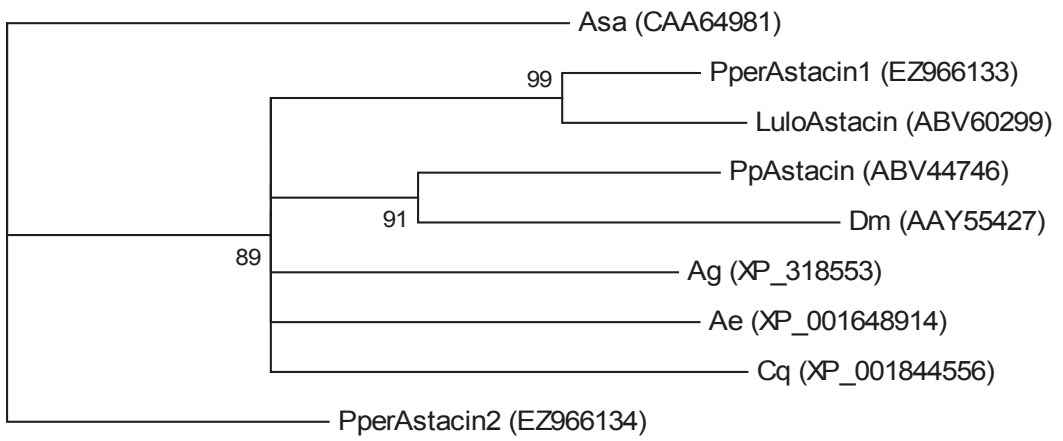

0.1

B

PperAstacin2
PperAstacin1
LuloAstacin
PpAstacin
D. melanogaster
An. gambiae
Ae. aegypti
C. quinque

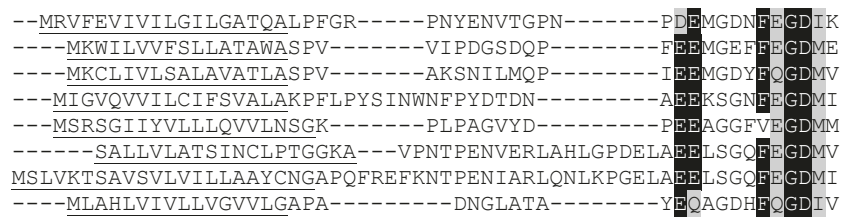
PperAstacin2 PperAstacin1 LuloAstacin PpAstacin D. melanogaster
An. gambiae Ae. aegypti C. quinque

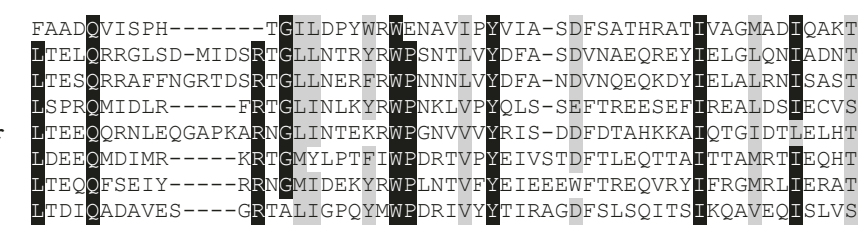
PperAstacin2 PperAstacin 1 LuloAstacin PpAstacin D. melanogaster An. gambiae Ae. aegypti Ae. aegypti
C. quinque
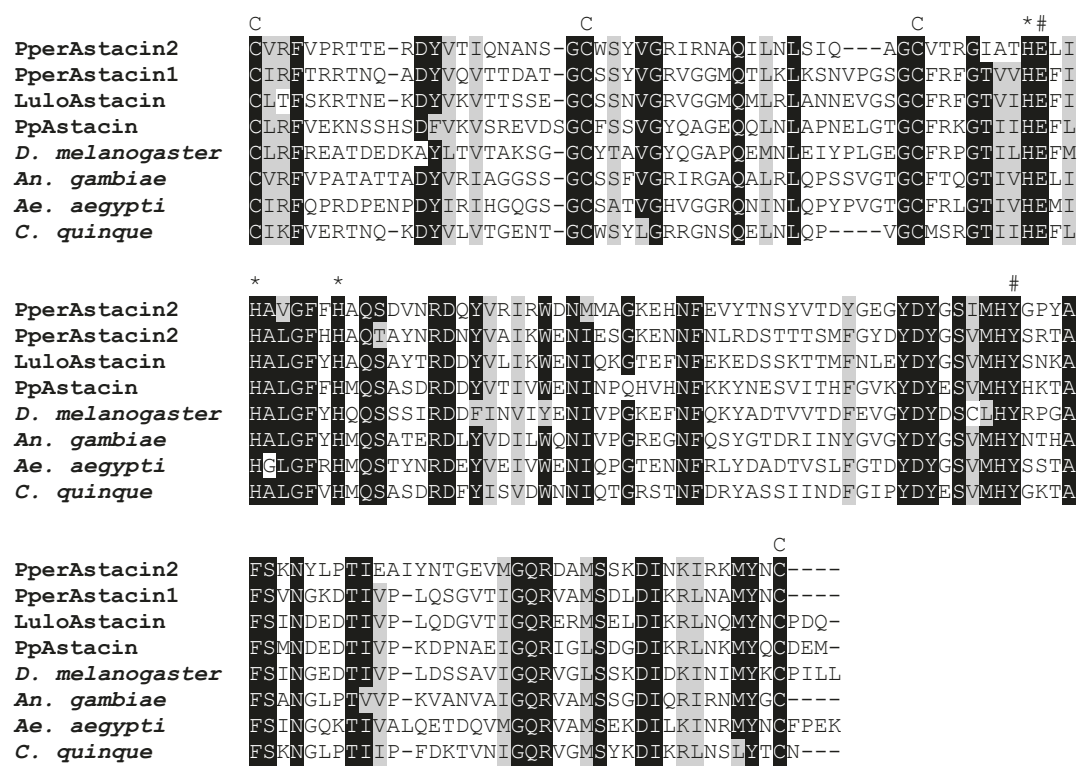

Figure 9 Phylogenetic analysis and sequence alignment of (putative) astacins. (A) Astacus astacus (Asa), Drosophila melanogaster (Dm), Aedes aegypti (Ae), Anopheles gambiae (Ag), Phlebotomus perniciosus (Pper), Lutzomyia longipalpis (Lulo), Phlebotomus papatasi (Pp) and Culex quinquefasciatus (Cq). Accession numbers are given in parentheses and node support is indicated by the bootstrap values. (B) Drosophila melanogaster (D. melanogaster), Aedes aegypti (A. aegypti), Anopheles gambiae (A. gambiae), Phlebotomus perniciosus (Pper), Lutzomyia longipalpis (Lulo), Phlebotomus papatasi (Pp) and Culex quinquefasciatus (C. quinque). Predicted signal peptide is underlined, conserved cysteines are indicated (C), the metal binding residues marked by $(*)$ and catalytic residues marked by (\#). Accession numbers: PperAstacin2 [GenBank: ABV44746], PperAstacin1 [GenBank:EZ966133], LuloAstacin [GenBank:ABV60299], PpAstacin [GenBank:ABV44746], D. melanogaster [GenBank: AAY55427], A. gambiae [GenBank:XP_318553], A. aegypti [GenBank:XP_001648914], C. quinque [GenBank:XP 001844556]. 
variants, sequence 5' truncated), PperMVP4 [GenBank: EZ933294] (cluster 139, 5' truncated) and PperMVP5 (EZ933295, cluster 52 and variants; $22 \mathrm{kDa}$, pI 4.77). PperMVP4 amino acid sequence, although truncated, contains predicted glycosylation sites (3 N-glycosylations). The only MVP transcript overrepresented in the sugar fed library is PperMVP3 [GenBank:EZ933293] (cluster 52 and variants, 5 ' truncated). As demonstrated in the phylogenetic analysis (Figure 10A), the identified sequences show high similarity to the respective five MVPs previously identified in the midgut of L. longipalpis. Homologs of four of these proteins are also known in P. papatasi (PpMVP1-4; Figure 10A). Interestingly, no sequence with high similarity to PperMVP5 was found in the midgut of $P$. papatasi. The phylogenetic tree also shows that PperMVP3 and its putative orthologues LuloMVP3 and PpMVP3 clade away from all the other sand fly and mosquito MVPs. This is in accordance with the fact that all the three seem to be downregulated be blood feeding unlike other MVPs. Multiple sequence alignment (Figure 10B) shows that the five putative $P$. perniciosus MVPs share little sequence homology suggesting that these molecules may have different functions altogether.

\section{Antimicrobial molecules}

Several transcripts encode proteins putatively involved in the immune response of the sand fly midgut. Two clusters coding for putative peptidoglycan recognition proteins (PGRPs) were identified. PGRPs play central and diverse roles in activating insect immune reactions including the melanization cascade, phagocytosis, and signal transduction pathways for production of antibacterial peptides. PperPGRPLB [GenBank:EZ617708], cluster 274 , is similar to a PGRP previously reported from L. longipalpis (LuloPGRP) [GenBank:ABV60332], and P. papatasi (PpPGRP) [GenBank:60369] midgut. Although the transcript appears to be 5 ' truncated, based on homology to other full length sand fly transcripts we predict it to be a protein of about $22 \mathrm{kDa}$. A homologous (but secreted) protein PGRPLB in Drosophila melanogaster [GenBank:AAN13505], mainly expressed in the midgut, was shown to regulate the Imd signalling pathway controlling the immune response against Gram-negative bacteria [31]. An A. gambiae PGRPLB homolog [GenBank:EAA01800] is induced by both bacterial and Plasmodium infections [32]. PperPGRPLC [GenBank:EZ617707], cluster 168, transcript 5' truncated encodes a putative protein with similarity to insect PGRPLC proteins that act as membrane-localized peptidoglycan receptors activating the Imd pathway. PGRPLC-like molecules have not been previously reported in sand flies. In searching the midgut transcriptomes of sand flies, partial transcripts with homology to
PperPGRPLC were found in P. papatasi [GenBank: ES347179] and L. longipalpis [GenBank:AM098991]. In A. gambiae, PGRPLC [GenBank:AGAP005203] signalling controls the size of symbiotic bacteria populations, intestinal bacterial infections and Plasmodium infections [33].

In addition to PGRPs, a transcript encoding a putative gram-negative bacteria binding protein was identified and named PperGNBP [GenBank:EZ617706], cluster 301 , transcript 5 ' truncated. Gram-negative bacteriabinding proteins serve as pattern recognition receptors binding to pathogen-associated beta-1,3-glucans in insects and they have been shown to play a role in mosquito defence against bacteria and Plasmodium infection [34].

It is likely that the identified sand fly pattern recognition proteins are involved in protection against bacteria in the midgut; however, similar to the mosquito homologs, they might also have an impact on Leishmania infection. Interestingly, Kumar et al. [35] have recently described a novel secreted peroxidase/dual oxidase system in A. gambiae midgut that catalyses cross-linking of a dityrosine network on the luminal surface of the epithelial cells. This network decreases the permeability of the mucus layer to immune elicitors and thus prevents induction of PGRPs, nitric oxide synthase and other immune responsive genes. Silencing of the peroxidase/dual oxidase system causes a drastic reduction in Plasmodium infection in the midgut [35]. We have not identified any homologs of the components of the peroxidase/dual oxidase system in P. perniciosus midgut in this analysis. Further studies are needed to see whether a dityrosine barrier is formed in the sand fly midgut. In our analysis, we did not detect any antimicrobial peptide transcripts in the midgut of $P$. perniciosus, although a defensin was previously characterized in Phlebotomus duboscqi midgut [36] and defensin transcripts were also reported from the midgut of L. longipalpis [6,7].

\section{Oxidative stress molecules}

A number of transcripts were identified coding for putative antioxidant enzymes. In hematophagous insects, blood meal-derived free heme is a strong pro-oxidant and can tax the midgut antioxidant system. In addition to their protective role, redox-related molecules were shown to regulate midgut epithelial immunity and impact the outcome of bacterial and parasitic infections in mosquitoes [35,37]

\section{Glutathione S-tranferases (GSTs) and peroxiredoxin}

Putative components of the glutathione-thioredoxin system, the central redox homeostasis maintaining pathway in insects, were found $[38,39]$. Several transcripts coding for putative glutathione-S-transferases (GSTs), enzymes 


\section{A}

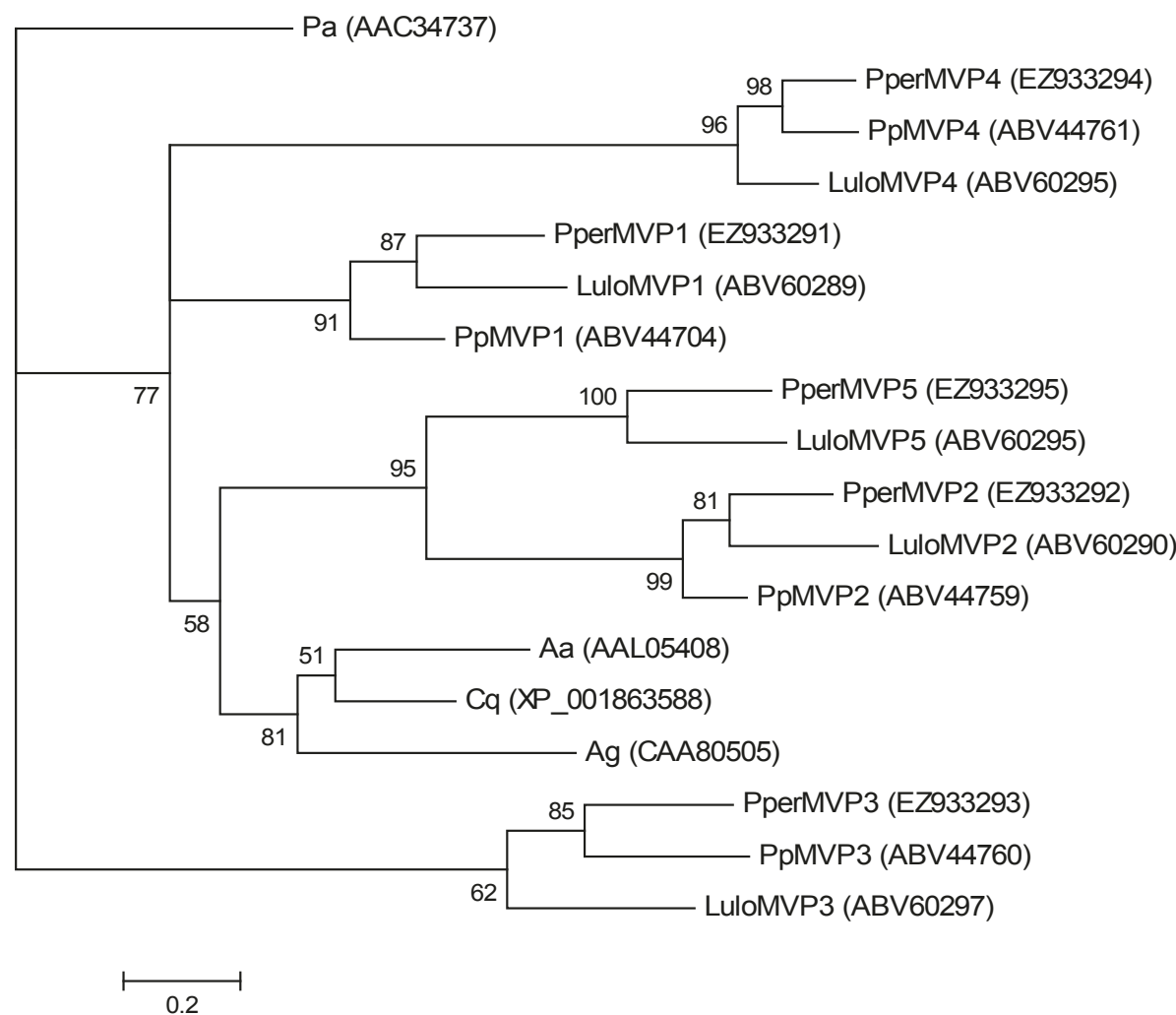

B

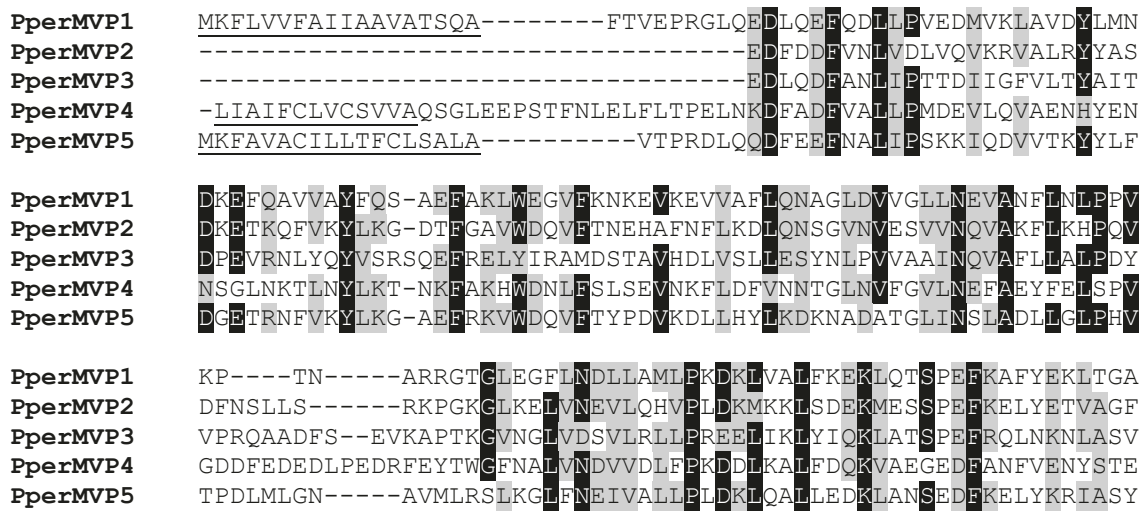

$\begin{array}{ll}\text { PperMVP1 } & \text { EFKKLTDSMFNSKEVQGYLKELKNHKVDLLKFLQVFKEFFGFP--- } \\ \text { PperMVP2 } & \text { DYEAMKDFVANSEEMLDLFETVRSYGLDLDGFFHQVEEFFGWN--- } \\ \text { PperMVP3 } & \text { ETAKAVSRVLFNQEVRRYSEVEKRGVNLVIAKQVVVYHLDL--- } \\ \text { PperMVP4 } & \text { EFRKLLKKLEMSPVAQKLFKRRKHGLDVYKLVNLGFLFGLN--- } \\ \text { PperMVP5 } & \text { DYCALEQETANSAEVQDFLNRLNGYGVSLDGLFNDVKEFFGWNCN }\end{array}$

Figure 10 Phylogenetic analysis and sequence alignment of putative microvillar proteins. (A) Periplaneta americana (Pa), Phlebotomus perniciosus (Pper), Phlebotomus papatasi (Pp), Lutzomyia longipalpis (Lulo), Aedes aegypti (Ae), Culex quinquefasciatus (Cq) and Anopheles gambiae (Ag). Accession numbers are given in parentheses. Node support is indicated by the bootstrap values. (B) Phlebotomus perniciosus (Pper). The predicted signal peptides are underlined. Accession numbers: PperMVP1 [GenBank:EZ933291], PperMVP2 [GenBank:EZ933292], PperMVP3 [GenBank:EZ933293], PperMVP4 [GenBank:EZ933294], PperMVP5 [GenBank:EZ933295]. 
catalyzing substrate detoxification by a thiol tripeptide glutathione, were identified in both libraries. PperGST1 [GenBank:EZ617709], cluster 163, encodes a putative intracellular GST of the Sigma subfamily. The putative protein is predicted to be $23.2 \mathrm{kDa}$ and have a $\mathrm{pI}$ of 5.00. It is nearly identical to putative Sigma GSTs described from the midgut of P. papatasi [GenBank: ABV44736] (98\% identity) and L. longipalpis LuloGST1 [GenBank:ABV60329] (97\% identity). PperGST2 [GenBank:EZ617710] (cluster 463, 5' truncated) encodes a putative transmembrane protein that has homology to microsomal GSTs of the MAPEG super family. Homologs of PperGST2 were found in the EST databases of $P$. papatasi [GenBank:FK811479] and L. longipalpis [GenBank:EW990920]. PperGST3 [GenBank:EZ617711] (cluster 1322, 5' truncated) shares similarity with other Dipteran GSTs of the Theta class, a class not previously reported in sand flies. A homolog of PperGST3 was identified by searching the L. longipalpis whole fly cDNA library [GenBank:AM099640] [40]. Unlike the midgut transcriptomes of $P$. papatasi and L. longipalpis, we have not found any GSTs of the Delta/Epsilon class, which may be due to the overall low abundance of the GST transcripts in sand fly midguts. Mosquito GSTs play an important role in as antioxidants and knockdown of GSTs of the Theta family has been reported to impact Plasmodium infections in A. gambiae and A. stephensi, although the effect varies with different parasitevector combinations [41].

A putative peroxiredoxin (or thioredoxin-dependent peroxidase), product of cluster 729, was identified and named PperPRX [GenBank:HM119220]. PperPRX encodes a putative intracellular protein of $16.7 \mathrm{kDa}$ and a pI of 7.1 containing a peroxiredoxin PRX5-like subfamily domain. Salp25D [GenBank:AF209911], a peroxiredoxin in the tick Ixodes scapularis, has been shown to facilitate the acquisition of Borrelia from an infected host by detoxifying reactive oxygen species at the vector-pathogen-host interface [42]. Midgut-specific Salp25D, while not significantly aiding the establishment of Borrelia, does have a slight protective effect. It is possible that sand fly peroxiredoxins, by detoxifying $\mathrm{OH}$ radicals, could have a similar protective effect on Leishmania parasites.

\section{Catalase and superoxide dismutases (SODs)}

Transcripts coding for putative enzymes of the superoxide dismutase (SOD)/catalase system were also identified. PperCat [GenBank:EZ617712], cluster 852, encodes a putative intracellular protein (molecular weight 57.7 $\mathrm{kDa}$ and $\mathrm{pI}$ 9.17) containing a catalase domain. It shares high similarity with a putative catalase molecule described in L. longipalpis midgut, LuloCat, [GenBank: ABV60342] and a similar sequence was also found in the $P$. papatasi midgut cDNA library [GenBank: ES351062]. Catalases are hydrogen peroxide detoxifying enzymes and for an A. gambiae homolog [GenBank: AGAP004904], expression is induced in the midgut after blood feeding in response to oxidative stress [43]. In the P. perniciosus midgut, the transcript was only found in the sugar fed library (9 sequences) and thus appears to be down regulated by blood feeding. A similar phenomenon could not be observed in L. longipalpis due to the low number of catalase sequences found (where one LuloCat transcript was found in the blood fed and one in the post-blood fed library infected with L. chagasi). The significance of PperCat down regulation by blood feeding remains unclear and post-transcriptional regulation cannot be excluded.

Two clusters with products containing copper-zinc superoxide dismutase (Cu-Zn SOD) domains were identified. PperSOD1 [GenBank:EZ617713], cluster 892, encodes a protein similar to a putative secreted $\mathrm{Cu}-\mathrm{Zn}$ SOD from the midgut of L. longipalpis, LuloSOD, [GenBank:ABV60343]. Despite the transcript being 5' truncated, based on homology to the Lutzomyia and mosquito molecules we predict the protein possesses a signal peptide and performs a similar function to LuloSOD. The molecule may be secreted or, given that the sequence contains a putative GPI-anchor site, GPIanchored to the plasma membrane of the midgut cells. PperSOD2 [GenBank:EZ617714], cluster 1166, encodes a putative intracellular protein (15.3 kDa, pI 6.3) similar to putative mosquito SODs. Intracellular SODs have not been previously described in the sand fly midgut, but when searched for homologous sequences, we found sequences coding for highly similar proteins in both $L$. longipalpis and $P$. papatasi midgut cDNA libraries [GenBank:EW987718 and GenBank:ES348811, respectively]. Phylogenetic analysis of mosquito and sand fly sequences (Figure 11) shows that extracellular and intracellular $\mathrm{Cu}-\mathrm{Zn}$ SODs form two distinct clades suggesting the two forms of the enzymes evolved prior to speciation of the two groups of organisms.

\section{Xanthine dehydrogenase}

Cluster 373 [GenBank:EZ617715], PperXDH, is a partial transcript coding for a product with high similarity to the C-terminal portion of L. longipalpis xanthine dehydrogenase (XDH) [GenBank:CAP08999.1]. XDHs catalyze the oxidation of xanthine to urate, the main product of nitrogen metabolism, which has antioxidant properties in insects. The XDH molecule has been shown to be up regulated after blood feeding in L. longipalpis. Silencing of L. longipalpis XDH resulted in a reduction in urate production and a reduced life span of both sugar fed and blood fed sand flies [44]. These results suggest that xanthine dehydrogenases are indeed 


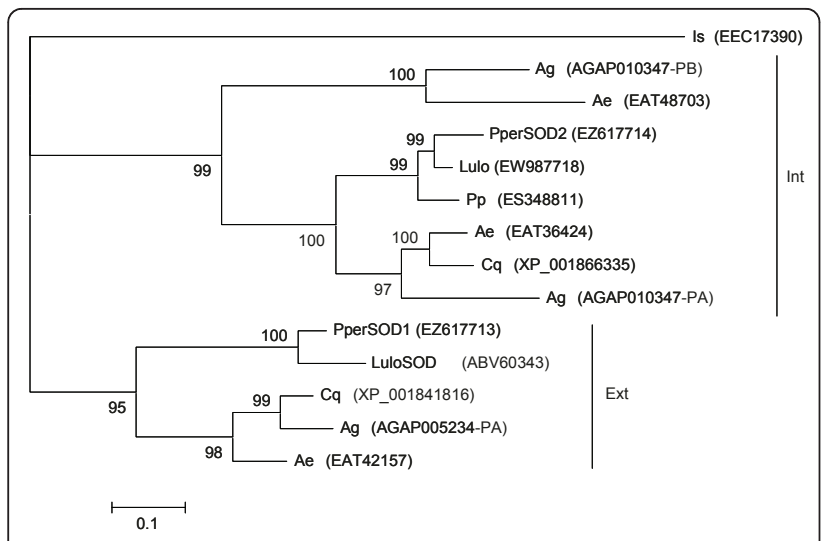

Figure 11 Phylogenetic analysis of superoxide dismutase molecules from Ixodes scapularis (Is), Anopheles gambiae (Ag), Aedes aegypti (Ae), Phlebotomus perniciosus (Pper), Lutzomyia longipalpis (Lulo), Phlebotomus papatasi (Pp) and Culex quinquefasciatus (Cq). GenBank accession numbers are given in parentheses, the clades are labelled with the respective localization based on SignalP prediction (Int: intracellular, Ext: extracellular) and node support is indicated by the bootstrap values.

involved in preventing oxidative damage by producing the antioxidant urate in sand flies.

\section{Ferritin}

Two molecules were identified with homology to ferritins described from other insect species including the sand flies $P$. papatasi and L. longipalpis. Similar to most insect ferritins, and unlike most vertebrate and plant ferritins, these molecules are likely secreted. PperFLC [GenBank:EZ617716], cluster 88, encodes a putative protein with homology to ferritin light-chain subunit and a molecular weight of $24.3 \mathrm{kDa}$ and pI 6.68, once secreted. PperFHC [GenBank:EZ617717], cluster 332, encodes a putative ferritin heavy-chain molecule. Although 5' truncated, PperFHC has high homology to P. papatasi and other species ferritin molecules with signal peptides and is likely similarly secreted. The transcripts originated in similar numbers from the blood fed and sugar fed libraries. Apart from their role in iron metabolism, these sand fly midgut ferritins may also play a role in preventing oxidative damage by sequestering large quantities of free iron from the digested blood meal as was suggested for other blood-feeding insects [45].

\section{Peritrophic matrix proteins and chitinase}

The peritrophic matrix (PM) is an extracellular chitincontaining matrix that is formed in the sand fly midgut after blood feeding that surrounds the ingested blood. Clusters coding for products with similarity to PM proteins described in other blood feeding Dipterans were identified. Three clusters coding for putative peritrophins were detected, originating in higher numbers from the blood fed library. These proteins share homology with molecules previously identified in other sand fly species and contain chitin binding domains (CBDs). PperPer1 [GenBank:EZ933302], cluster 97, encodes a protein similar to putative peritrophins with four CBDs previously described from the midgut of $L$. longipalpis (LuloPer1) [GenBank:ABV60306] and P. papatasi (PpPer1) [GenBank:ABV44705]. PperPer1 is represented by 94 sequences found only in the blood fed library. Although PperPer1 ESTs appears to be incomplete at the 5 ' end and missing the first $13 \mathrm{~N}$-terminal amino acids, based on homology, we predict it to be a secreted molecule of mature molecular weight of $28.2 \mathrm{kDa}$ and pI 4.68. PperPer2 [GenBank:HM119221], cluster 330, encodes a putative peritrophin with similarity to a $L$. longipalpis protein LuloPer2 [GenBank:ABV60320]containing one CBD. A third putative peritrophin, PperPer3 [GenBank:HM119222], cluster 156, shows similarity to a P. papatasi peritrophin PpPer3 [GenBank:ABV44751] and contains two putative CBDs (one partial CBD sequenced due to a 5 ' truncation). Phylogenetic analysis (Figure 12) of the CBDs from $P$. perniciosus, $P$. papatasi and $L$. longipalpis illustrates a high degree of conservation of the Peritrophin 1 arrangement. The four peritrophin domains share respective homology in all the three sand fly species.

A cluster coding for a putative chitinase was identified in the blood fed library. The molecule, named PperChit

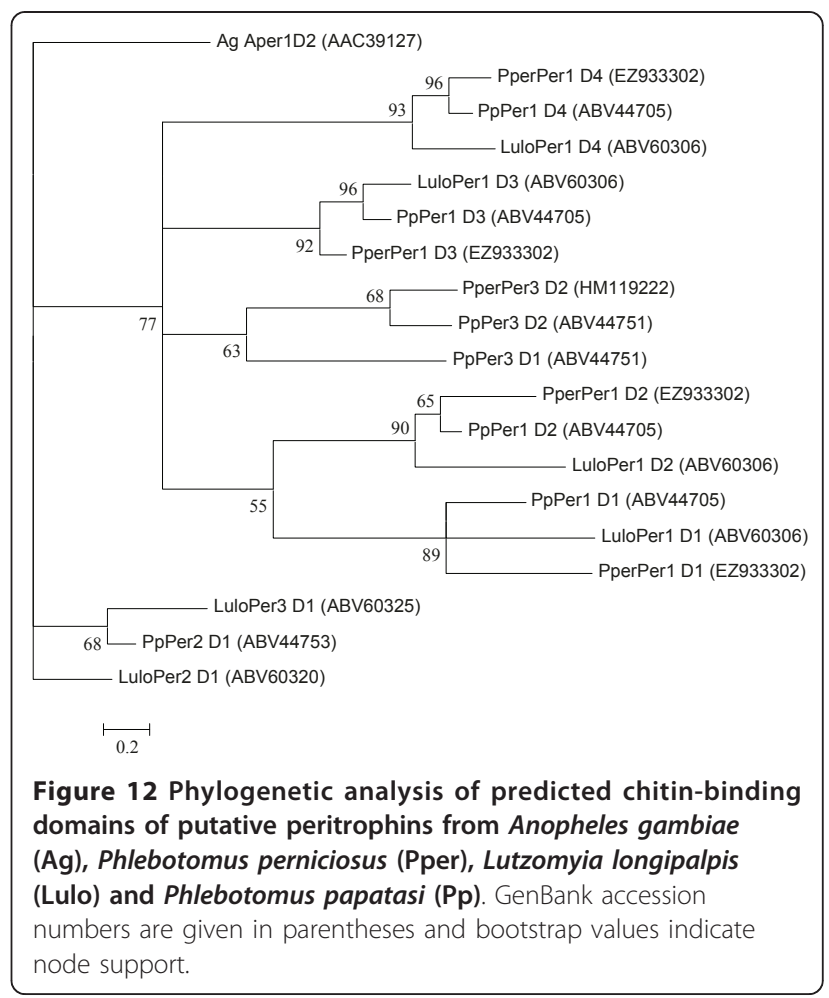


[GenBank:EZ933285] (cluster 124) encodes a putative protein containing a CBD and a mucin-like domain and shares high similarity to $P$. papatasi PpChit1 [GenBank: AAV49322] and L. longipalpis LlChit1 [GenBank: AAN71763] chitinases. These midgut-specific, blood meal-induced enzymes have been shown to account for chytinolytic activity in the sand fly midgut and have been implicated in the release of Leishmania parasites from the endoperitrophic space $[8,46,47]$.

Along with peritrophins and chitinases, non-chitin binding peritrophic matrix proteins have also been described from bloodsucking insects. Dinglasan et al. [48] performed a proteomic analysis of A. gambiae peritrophic matrix and identified a number of non-chitinbinding proteins including proteolytic enzymes and novel classes of PM proteins with unknown function. We identified clusters with homology to these proteins in the P. perniciosus libraries. The product of cluster 379 [GenBank:EZ933287] is highly similar to A. gambiae PM protein [GenBank:AGAP006398]. The transcript contains a potential N-glycosylation site and 3 DM9 repeats (repeats of unknown function found in a number of arthropod proteins). A homolog was found in the P. papatasi midgut library (31.5 kDa midgut protein), [GenBank:ABV44721]. The product of cluster 358 [GenBank:EZ933286] (5' truncated) showed a significant similarity (BLASTp match $\mathrm{E}=4 \mathrm{e}-18$ ) to an $A$. gambiae PM protein [GenBank:AGAP000570]. The sequences originated from both blood fed (3 sequences) and sugar fed (10 sequences) libraries. Homologous proteins were also found in the midgut of P. papatasi [GenBank: ABV44744] and L. longipalpis [GenBank:ABV60298] and also the salivary glands of $P$. duboscqi [GenBank: ABI20163]. These proteins contain no described conserved domains. Based on homology to the A. gambiae protein found in the PM, we speculate that the product of cluster 358 may also be involved in the PM formation in sand flies. On the other hand, its expression in the sugar fed midgut as well as the presence of homologs in the salivary glands may suggest a different function for this protein, such as regulating the haemostatic response.

The structure of the mosquito and sand fly peritrophic matrix is complex and rearranges during the course of blood digestion [49]. The two putative peritrophins with multiple CBDs (PperPer1 and PperPer3) are likely to have a role in cross-linking the chitin fibrils of the peritrophic matrix. In addition to chitin binding, mosquito proteins with CBDs have also been described to bind heme and have a role in its sequestration during blood digestion [50]. Also, the glycosylation of the PM proteins can be of great importance for the PM structure and function. Two of the putative peritrophins, PperPer2 and PperPer3, and the putative chitinase, PperChit, contain mucin-like (Pro-Ser/Thr rich) domains. Glycosylation of these domains can influence the selectiveness of the PM pores, account for water retention within the PM and also protect the molecules from degradation by proteolytic enzymes. Furthermore, the degradation of putatively aglycosylated PM proteins (like PperPer1 and the product of cluster 358) by temporally secreted digestive proteases may play a role in the changes in the PM thickness and structure.

\section{Transcripts differentially expressed after blood feeding}

In order to identify changes in expression of midgut proteins induced by blood feeding, we compared the abundance of transcripts in the sugar fed and the blood fed libraries using chi-square statistical analysis. We found several transcripts that were significantly more abundant after blood feeding and several that were underrepresented in the blood fed library (see tables 3 and 4). As expected, we observed some transcripts putatively involved in blood digestion and peritrophic matrix formation more abundant after blood feeding. These included the putative peritrophin with four chitin-binding domains, PperPer1. Similar to the putative orthologues in $P$. papatasi and L. longipalpis, PpPer1 [GenBank:EU031912] and LuloPer1 [GenBank: EU124588], PperPer1 was only detected in the blood fed midgut library. With regard to peritrophin sequence abundance, it is interesting to note that we did not detect any peritrophin sequence highly represented before blood feeding. This is in contrast with the situation described in P. papatasi, where high numbers of a putative peritrophin with one chitin binding domain, PpPer2 [GenBank:EU047543], were detected in sugar fed midguts. In this respect, the observed profile is more similar to peritrophins in the midgut of a more distantly related species L. longipalpis.

Transcripts coding for proteolytic enzymes, namely the chymotrypsins PperChym1 and PperChym 2 and the trypsin PperTryp3, were also found more abundant in the blood fed library and thus likely represent digestive

Table 3 ESTs overrepresented in the blood fed library (BF) in comparison to the sugar fed library (SF)

\begin{tabular}{lllll}
\hline Cluster \# & Putative function & SF & BF & P value \\
\hline 45 & microvillar protein (PperMVP1) & 0 & 681 & $7.49 \mathrm{E}-185$ \\
97 & peritrophin (PperPer1) & 0 & 94 & $4.46 \mathrm{E}-23$ \\
81 & chymotrypsin (PperChym1) & 0 & 82 & $2.83 \mathrm{E}-20$ \\
52 & microvillar protein (PperMVP5) & 0 & 35 & $2.10 \mathrm{E}-09$ \\
63 & trypsin (PperTryp3) & 0 & 31 & $1.76 \mathrm{E}-08$ \\
139 & microvillar protein (PperMVP4) & 0 & 28 & $8.67 \mathrm{E}-08$ \\
40 & microvillar protein (PperMVP2) & 0 & 26 & $2.52 \mathrm{E}-07$ \\
79 & unknown (lipid recognition) & 0 & 9 & $2.47 \mathrm{E}-03$ \\
102 & chymotrypsin (PperChym2) & 2 & 11 & $1.14 \mathrm{E}-02$ \\
\hline
\end{tabular}


Table 4 ESTs overrepresented in the sugar fed library (SF) in comparison to the blood fed library (BF)

\begin{tabular}{lllll}
\hline Cluster \# & Putative function & SF & BF & P value \\
\hline 46 & trypsin (PperTryp1) & 513 & 20 & $3.16 \mathrm{E}-115$ \\
249 & unknown & 25 & 3 & $3.60 \mathrm{E}-05$ \\
1033 & chymotrypsin (PperChym3) & 12 & 0 & $5.76 \mathrm{E}-04$ \\
16 & trypsin (PperTryp2) & 10 & 0 & $1.68 \mathrm{E}-03$ \\
852 & catalase (PperCat) & 10 & 0 & $2.88 \mathrm{E}-03$ \\
174 & glycoside hydrolase (PperGH13) & 23 & 9 & $1.46 \mathrm{E}-02$ \\
18 & microvillar protein (PperMVP3) & 18 & 6 & $1.56 \mathrm{E}-02$ \\
652 & 40S ribosomal protein SA & 8 & 1 & $2.07 \mathrm{E}-02$ \\
183 & glycoside hydrolase (PperGH31) & 12 & 3 & $2.16 \mathrm{E}-02$ \\
\hline
\end{tabular}

enzymes induced by the intake of blood. On the other hand, three other putative proteases, PperTryp1, PperTryp 2 and PperChym 3 were significantly less abundant in the blood fed library. We speculate that these molecules may be post-transcriptionally regulated digestive enzymes that are stored in the midgut prior to blood feeding.

The most striking differences in sequence abundance before and after blood feeding were observed for the microvillar proteins. Four of the five identified putative microvillar proteins (PperMVP1, 2, 4 and 5) were only detected in the blood fed library and in high abundance. This indicates a strong up-regulation of these proteins after the intake of blood. In contrast, PperMVP3 was overrepresented in the sugar fed library, suggesting a different role for this protein. The observed microvillar proteins EST distributions are in accordance with what was described in both P. papatasi and L. longipalpis, where the PperMVP3 orthologues, LuloMVP3 and $P p M V P 3$ were highly represented before blood feeding unlike all other microvillar proteins. The conservation of these proteins and their pattern of expression in the midgut of the three sand fly species indicate their important, yet uncharacterized, roles in the midgut physiology.

The list of sequences overabundant in the blood fed library also includes a putative protein, cluster 79 [GenBank:HQ015441], similar to a putative cockroach allergen MPA2 and several uncharacterized mosquito proteins. The presence of a lipid-binding ML domain in the translated sequence of cluster 79 [Interpro: IPR003172] may suggest a role of lipid recognition. In accordance with their putative function in carbohydrate digestion, putative glycoside hydrolases, cluster 174 [GenBank:HQ015444] and cluster 183 [GenBank: HQ015443], were found overrepresented in the sugar fed library. Interestingly, we also found a putative catalase sequence (PperCat) overabundant in the sugar fed midgut. Similarly, the significance of the higher abundance of a putative $40 \mathrm{~S}$ ribosomal protein SA (cluster
652) [GenBank:HQ015442] and an unknown, probably non-coding, sequence (cluster 249) [GenBank: GW817178] represented by the clone in the sugar fed midgut, remains unknown.

\section{Conclusion}

P. perniciosus is a medically important vector of canine and human visceral leishmaniasis in the Old World. To date, the only molecular data available for this species have been the salivary gland transcripts [51]. This study is the first report on molecules present in the midgut of P. perniciosus. As development of Leishmania in the vector sand fly is restricted to the digestive tract, the midgut is the primary organ where interactions with Leishmania take place. By sequencing and analyzing transcripts present before and after blood feeding, we have provided a catalogue of putative proteins potentially involved in feeding and blood digestion. All the generated ESTs were deposited in the NCBI dbEST database, making them available to scientific communities for further research. Selected molecules of interest were manually annotated and the nucleotide and putative protein sequences submitted to GenBank.

We have identified a variety of molecules, including putative proteins that have not been previously described in the sand fly midgut. Among the putative proteases, these include a putative astacin (PperAstacin2) and a putative chymotrypsin (PperChym5). We also found molecules potentially involved in pathogen recognition such as the gram-negative bacteria binding protein (PperGNBP) and the putative peptidoglycan receptor (PperPGRPLC). Novel putative antioxidant enzymes were also identified including an intracellular superoxide dismutase (PperSOD2) and putative microsomal and Theta class glutathione S-transferases (PperGST2 and PperGST3). In addition, we describe homologs of mosquito peritrophic matrix proteins.

Constructing libraries from sand fly females before and after the intake of blood allowed for the identification of molecules differentially expressed in response to blood feeding. By comparing our findings with the midgut transcriptome analyses of two other sand fly species, L. longipalpis and P. papatasi, we identified several features shared by the two permissive vectors, $P$. perniciosus and $L$. longipalpis. These include the absence of a significant number of peritrophin sequences before blood feeding, while in P. papatasi, a peritrophin with one chitin binding domain was abundant in sugar fed midguts.

Altogether, this study contributes to our knowledge of the molecular background of events that occur in the sand fly midgut. It provides a valuable platform for functional studies of selected molecules relevant in the transmission of Leishmania. These may represent 
targets for use as novel vector-based transmission-blocking vaccines to control this neglected disease.

\section{Methods}

\section{Sand fly maintenance and dissection}

The colony of Phlebotomus perniciosus (originally from Spain) was maintained in the insectary of Charles University in Prague as described previously [52]. Adults were kept at $26^{\circ} \mathrm{C}$ and fed on $50 \%$ sucrose ad libitum. Ten midguts from 3-5 days old sugar fed only females were dissected for the sugar fed library construction. Females were fed on an anaesthetised Balb/C mouse and two midguts containing blood were dissected at each of the following time points: 4-6 h, 24 h, 2 days, 3 days and 4 days post-blood meal. These samples were pooled for the construction of the blood fed library. For the qPCR experiment, females were fed through a chick skin on heat-inactivated rabbit blood containing $L$. infantum infected macrophages (or uninfected macrophages in the control group). Midguts from ten infected and ten uninfected (control) females were dissected $6 \mathrm{~h}$, $24 \mathrm{~h}, 72 \mathrm{~h}$ and 10 days after blood feeding and stored individually in $10 \mu \mathrm{l}$ of RNAlater (Ambion) as well as 10 individual midguts from sand flies before blood feeding (sugar fed). Presence of promastigotes in midguts was confirmed $72 \mathrm{~h}$ after blood feeding. On day 10 after blood feeding only sand flies with late-stage infections (with parasites on the stomodeal valve) were used.

\section{cDNA library construction and sequencing}

Messenger RNA was purified from midguts stored in RNAlater (Ambion) using the MicroFastTrack mRNA isolation kit (Invitrogen). PCR-based cDNA libraries were prepared following the instructions for the SMART cDNA library construction kit (Clontech). Each cDNA library was then fractionated into three sets containing small, medium and large fragments using columns provided by the manufacturer. Concentrated cDNA was ligated into a lambda TriplEx2 vector (Clontech). The resulting ligation reaction was packed using the Gigapack III Gold (Stratagene). The libraries thus obtained were plated by infecting log-phase XL1-blue cells (Clontech). Phage plaques lacking $\beta$-galactosidase activity were picked using sterile wooden sticks and placed into $75 \mu \mathrm{l}$ of water. Amplification of the cDNA was performed using Faststart Mix (Roche), $3 \mu \mathrm{l}$ template and primers PT2F1 (5'-AAG TAC TCT AGC AAT TGT GAG C-3') and PT2R1 (5'-CTC TTC GCT ATT ACG CCA GCT G-3'). The PCR conditions were 1 hold of $75^{\circ} \mathrm{C}$ for $3 \mathrm{~min}, 1$ hold of $94^{\circ} \mathrm{C}$ for $4 \mathrm{~min}, 33$ cycles of $94^{\circ} \mathrm{C}$ for $1 \mathrm{~min}, 49^{\circ} \mathrm{C}$ for $1 \mathrm{~min}$, and $72^{\circ} \mathrm{C}$ for 7 min, 1 hold of $75^{\circ} \mathrm{C}$ for $7 \mathrm{~min}$. The amplification product was cleaned with three washes with ultra pure water using ExcelaPure plates (EdgeBio) resuspended in
$30 \mu \mathrm{l}$ of water. Sequencing was performed at the Rocky Mountain Laboratories Genomics Unit as described previously [53]. The template was combined with primer PT2F3 (5' - TCT CGG GAA GCG CGC CAT TGT-3') in an ABI 96-well Optical Reaction Plate (P/N 4306737) following the manufacturers recommendations. Sequencing reactions were setup as recommended by Applied Biosystems BigDye Terminator v3.1 Cycle Sequencing Kit by adding $1 \mu \mathrm{L}$ ABI BigDye Terminator Ready Reaction Mix v3.1 (P/N 4336921), $1.5 \mu \mathrm{L} 5 \times$ ABI Sequencing Buffer (P/N 4336699), and $3.5 \mu \mathrm{L}$ of water for a final volume of $10 \mu \mathrm{L}$. Cycle sequencing was performed at $96^{\circ} \mathrm{C}$ for $10 \mathrm{~s}, 50^{\circ} \mathrm{C}$ for $5 \mathrm{~s}, 60^{\circ} \mathrm{C}$ for $4 \mathrm{~min}$ for 27 cycles on either a Bio-Rad Tetrad 2 (Bio-Rad Laboratories, Hercules, CA) or ABI 9700 (Applied Biosystems, Inc., Foster City, CA) thermal cycler. Fluorescently labelled extension products were purified following Applied Biosystems BigDye XTerminator Purification protocol and then processed on an ABI 3730xL DNA Analyzer (Applied Biosystems, Inc., Foster City, CA).

\section{Bioinformatic analysis}

The bioinformatic analyses of the sequences were performed using the dCAS 1.4 cDNA annotation software [14]. Briefly, primer, vector and low quality sequences were removed at the 5' and 3' ends of each sequence using Cap3 and Phred software [54-56]. Sequences from both libraries were grouped together and aligned to generate clusters based on 95\% identity over 100 nucleotides. Three frame translated consensus sequences were supplied to the appropriate BLAST algorithm [57] for comparison to the contents of the NCBI non-redundant protein database, the Gene Ontology database [58], the KOG conserved domain database [59], Simple Modular Architecture Tool (SMART) [60], Protein Family Database (Pfam) [61], rRNA subset database and Mitochondrial and Plasmid Sequences database (MIT-PLA) available from NCBI. The predicted presence of a signal secretion peptide or transmembrane domains was determined using the SignalP and TMHMM programs respectively $[62,63]$. $\mathrm{N}$ - and $\mathrm{O}$-glycosylation site prediction was performed for selected sequences using NetNGlyc 1.0 and NetOGlyc 3.1 software http://www.cbs.dtu. $\mathrm{dk} /$ services/NetNGlyc/[64]. Numbers of sequences in the sugar fed and the blood fed library were compared using $\chi^{2}$ statistical analysis. Clusters with significantly unequal distribution of the clone sequences $(\mathrm{P}<0.05$ and expected frequency in each of the libraries $>4$ ) were identified as over- or underrepresented after blood feeding. Selected sequences were aligned using Clustal $\times$ 2.0 [65] and manually refined in BioEdit 7.0 sequenceediting software. For phylogenetic analyses of amino acid sequences, best substitution matrix was determined for each alignment by ProtTest software, version 2.0 
[66]. This matrix was then used by TREE-PUZZLE 5.2 [67] to reconstruct maximum likelihood phylogenetic trees from the protein alignments using quartet puzzling with 1000 puzzling steps. Resulting trees were visualized in MEGA 4 [68].

\section{Macrophage infection}

Leishmania infantum (MCAN/PT/05/IMT 373) parasites were cultured at $23^{\circ} \mathrm{C}$ in RPMI medium (Sigma) containing $10 \%$ heat-inactivated foetal calf serum (FCS, Gibco), $50 \mathrm{ug} / \mathrm{ml}$ gentamicin, $1 \times$ BME vitamins (Sigma) and $1 \%$ human urine. Mouse macrophage line J774 was cultured at $37^{\circ} \mathrm{C}, 5 \% \mathrm{CO}_{2}$ in RPMI medium containing $10 \%$ FCS, $2 \mathrm{mM}$ alanyl-glutamine and penicillin (200 $\mathrm{IU} / \mathrm{ml}$ ). Macrophages were infected with stationaryphase $L$. infantum parasites at 1:10 macrophage:parasite ratio. After $24 \mathrm{~h}$ of co-cultivation at $37^{\circ} \mathrm{C}, 5 \% \mathrm{CO}_{2}$, noninternalized parasites were removed by washing $3 \times$ in the culture media and infected macrophages cultivated overnight. Macrophages were confirmed to contain amastigotes by light microscopy of Giemsa-stained slides. The infected macrophage culture was scraped from the culture plates, centrifuged at $380 \mathrm{~g}$ for $10 \mathrm{~min}$ utes and resuspended in heat-inactivated rabbit blood for sand fly infections at the concentration of $3 \times 10^{6}$ macrophages $/ \mathrm{ml}$.

\section{Quantitative PCR}

RNA was purified from individual midguts stored in $-20^{\circ} \mathrm{C}$ using a High Pure RNA Tissue Kit (Roche) and cDNA synthesized using SuperScript III Reverse Transcriptase (Invitrogen) with random hexamer primers (Promega) following manufacturer's instructions. Quantification of putative trypsin transcripts was performed by real-time PCR with primers designed for PperTryp1 (5'-CCC AAT GGA CTA TGA CTA CGC-3' and 5'CGA ACA TCG TCG AAT ACG ATA G-3'), PperTryp2 (5'-GGT GTT CTC GTT GGA GTG GT-3' and 5'-TGG CGT AAA CTC CAG GGT AG-3'), PperTryp3 (5'-TGA GGA TGT TGA GGA TGG AA-3' and 5'CTC TTG GTT ATT CAG AGT GTT ACC C-3') and PperS7 ribosomal protein as a reference transcript (5'ATC CCT ATG CCG AAG CAG A-3' and 5'-TCA AGC TCA CGT ACC AGA CG-3'). The amplification reaction was carried out using the iQ5 real-time PCR detection system (Bio-Rad) by using the SYBR Green detection method (iQ SYBR Green Supermix, Bio-Rad) in $15 \mu \mathrm{l}$ reaction volume containing $1 \mu \mathrm{l}$ cDNA template and $0.5 \mu \mathrm{l}$ primer set ( $5 \mathrm{uM}$ each). The running conditions were as follows: $3 \mathrm{~min}$ at $95^{\circ} \mathrm{C}$ followed by 40 repetitive cycles: $10 \mathrm{~s}$ at $95^{\circ} \mathrm{C}, 10 \mathrm{~s}$ at $55^{\circ} \mathrm{C}$, and $10 \mathrm{~s}$ at $72^{\circ} \mathrm{C}$. Reactions were run in duplicates and data were analysed using the $2^{-\triangle \Delta C T}$ (Livak) method. In a preliminary experiment we have established that the target genes (trypsins) and the reference gene (PperS7 ribosomal protein) have similar (about 5\% variance) amplification efficiencies which are nearly $100 \%$. Data are presented as relative transcript levels using the $\mathrm{S} 7$ ribosomal protein gene as an internal control. Statistical analysis was done by Mann-Whitney U Test using (Statistica 6.1 StatSoft software).

\section{Acknowledgements and Funding}

This work was supported by the Intramural Research Program of the Division of Intramural Research, National Institute of Allergy and Infectious Diseases, National Institutes of Health, the Wellcome trust project 078937, Czech Science Foundation (projects 206/09/0777 and 206/09/H026), Czech Ministry of Education (projects MSM0021620828 and LC06009) and the Boehringer Ingelheim Fonds. The content of this publication does not necessarily reflect the views or policies of the Department of Health and Human Services, nor does mention of trade names, commercial products, or organization imply endorsement by the government of the United States of America.

\section{Author details}

'Department of Parasitology, Faculty of Science, Charles University in Prague, Vinicna 7, 12844 Praha 2, Czech Republic. ${ }^{2}$ Genomics Unit, Research Technologies Section, Rocky Mountain Laboratories, Hamilton, Montana 59840, USA. ${ }^{3}$ Vector Molecular Biology Unit, Laboratory of Malaria and Vector Research, NIAID, NIH, Rockville, MD, 20852, USA.

\section{Authors' contributions}

AD participated in the study design, sand fly rearing, dissections, CDNA library construction and annotation, sequence alignment, phylogenetic analysis, quantitative PCR and drafting the manuscript. AJF and KDB sequenced all cDNA amplification products selected from the library. JV participated in the quantitative PCR experiment. PV and JGV participated in the study design and coordination, and revised the manuscript. RCJ conceived the study, participated in its design and coordination and revised the manuscript. All authors read and approved the final manuscript.

Received: 17 November 2010 Accepted: 10 May 2011

Published: 10 May 2011

\section{References}

1. Killick-Kendrick R: The biology and control of phlebotomine sand flies. Clin Dermatol 1999, 17:279-289.

2. Kamhawi S: Phlebotomine sand flies and Leishmania parasites: friends or foes? Trends Parasitol 2006, 22:439-445.

3. Peacock CS, Seeger K, Harris D, Murphy L, Ruiz JC, Quail MA, Peters N, Adlem E, Tivey A, Aslett M, Kerhornou A, Ivens A, Fraser A, Rajandream MA, Carver T, Norbertczak H, Chillingworth T, Hance Z, Jagels K, Moule S, Ormond D, Rutter S, Squares R, Whitehead S, Rabbinowitsch E, Arrowsmith C, White B, Thurston S, Bringaud F, Baldauf SL, Faulconbridge A, Jeffares D, Depledge DP, Oyola SO, Hilley JD, Brito LO, Tosi LR, Barrell B, Cruz AK, Mottram JC, Smith DF, Berriman M: Comparative genomic analysis of three Leishmania species that cause diverse human disease. Nat Genet 2007, 39:839-847.

4. Oliveira F, Jochim RC, Valenzuela JG, Kamhawi S: Sand flies, Leishmania, and transcriptome-borne solutions. Parasitol Int 2009, 58:1-5.

5. Ramalho-Ortigao M, Jochim RC, Anderson JM, Lawyer PG, Pham VM, Kamhawi S, Valenzuela JG: Exploring the midgut transcriptome of Phlebotomus papatasi: comparative analysis of expression profiles of sugarfed, blood-fed and Leishmania major-infected sandflies. BMC Genomics 2007, 8.

6. Jochim RC, Teixeira CR, Laughinghouse A, Mu JB, Oliveira F, Gomes RB, Elnaiem DE, Valenzuela JG: The midgut transcriptome of Lutzomyia longipalpis: comparative analysis of CDNA libraries from sugar-fed, blood-fed, post-digested and Leishmania infantum chagasi-infected sand flies. BMC Genomics 2008, 9.

7. Pitaluga AN, Beteille V, Lobo AR, Ortigao-Farias JR, Davila AM, Souza AA, Ramalho-Ortigão JM, Traub-Cseko YM: EST sequencing of blood-fed and 
Leishmania-infected midgut of Lutzomyia longipalpis, the principal visceral leishmaniasis vector in the Americas. Mol Genet Genomics 2009, 282:307-317.

8. Ramalho-Ortigao JM, Kamhawi S, Joshi MB, Reynoso D, Lawyer PG, Dwyer DM, Sacks DL, Valenzuela JG: Characterization of a blood activated chitinolytic system in the midgut of the sand fly vectors Lutzomyia longipalpis and Phlebotomus papatasi. Insect Mol Biol 2005, 14:703-712.

9. Kamhawi S, Ramalho-Ortigao M, Pham VM, Kumar S, Lawyer PG, Turco SJ, Barillas-Mury C, Sacks DL, Valenzuela JG: A role for insect galectins in parasite survival. Cell 2004, 119:329-341.

10. Sant'Anna MR, Diaz-Albiter H, Mubaraki M, Dillon RJ, Bates PA: Inhibition of trypsin expression in Lutzomyia longipalpis using RNAi enhances the survival of Leishmania. Parasit Vectors 2009, 2:62.

11. Pimenta PFP, Saraiva EMB, Rowton E, Modi GB, Garraway LA, Beverley SM, Turco SJ, Sacks DL: Evidence that the vectorial competence of phlebotomine sand flies for different species of Leishmania is controlled by structural polymorphisms in the surface lipophosphoglycan. Proc Natl Acad Sci USA 1994, 91:9155-9159.

12. Myskova J, Svobodova M, Beverley SM, Volf P: A lipophosphoglycanindependent development of Leishmania in permissive sand flies. Microbes Infect 2007, 9:317-324

13. Svarovska A, Ant TH, Seblova V, Jecna L, Beverley SM, Volf P: Leishmania major glycosylation mutants require phosphoglycans (lpg2-) but not lipophosphoglycan (lpg1-) for survival in permissive sand fly vectors. PLoS Negl Trop Dis 2010, 4:e580.

14. Guo Y, Ribeiro JM, Anderson JM, Bour S: dCAS: a desktop application for cDNA sequence annotation. Bioinformatics 2009, 25:1195-1196.

15. Ramalho-Ortigao JM, Kamhawi S, Rowton ED, Ribeiro JMC, Valenzuela JG: Cloning and characterization of trypsin- and chymotrypsin-like proteases from the midgut of the sand fly vector Phlebotomus papatasi. Insect Biochem Mol Biol 2003, 33:163-171.

16. Telleria EL, Pitaluga AN, Ortigao-Farias JR, de Araujo APO, RamalhoOrtigao JM, Traub-Cseko YM: Constitutive and blood meal-induced trypsin genes in Lutzomyia longipalpis. Arch Insect Biochem Physiol 2007, 66:53-63.

17. Dillon RJ, Lane RP: Bloodmeal Digestion in the Midgut of Phlebotomus papatasi and Phlebotomus langeroni. Med Vet Entomol 1993, 7:225-232.

18. Schlein Y, Romano H: Leishmania major and L. donovani: effects on proteolytic enzymes of Phlebotomus papatasi (Diptera: Psychodidae). Exp Parasitol 1986, 62:376-380.

19. Dillon RJ, Lane RP: Influence of Leishmania Infection on Blood-Meal Digestion in the Sandflies Phlebotomus-papatasi and P. langeroni. Parasitol Res 1993, 79:492-496.

20. Telleria EL, de Araujo AP, Secundino NF, vila-Levy CM, Traub-Cseko YM: Trypsin-Like Serine Proteases in Lutzomyia longipalpis - Expression, Activity and Possible Modulation by Leishmania infantum chagasi. PLoS One 2010, 5:e10697.

21. Graf $R$, Briegel $H$ : The synthetic pathway of trypsin in the mosquito Aedes aegypti L (Diptera, Culicidae) and in vitro stimulation in isolated midguts. Insect Biochem 1989, 19:129-137.

22. Titani $K$, Ericsson $L H$, Walsh KA, Neurath $H$ : Amino-acid sequence of bovine carboxypeptidase B. Proc Natl Acad Sci USA 1975, 72:1666-1670

23. Lavazec C, Boudin C, Lacroix R, Bonnet S, Diop A, Thiberge S, Boisson B, Tahar R, Bourgoin C: Carboxypeptidases B of Anopheles gambiae as targets for a Plasmodium falciparum transmission-blocking vaccine. Infect Immun 2007, 75:1635-1642.

24. Dinglasan RR, Kalume DE, Kanzok SM, Ghosh AK, Muratova O, Pandey A4, et al: Disruption of Plasmodium falciparum development by antibodies against a conserved mosquito midgut antigen. Proc Natl Acad Sci USA 2007, 104:13461-13466.

25. Abdullah MA, Valaitis AP, Dean DH: Identification of a Bacillus thuringiensis Cry11Ba toxin-binding aminopeptidase from the mosquito, Anopheles quadrimaculatus. BMC Biochem 2006, 7:16.

26. Pomes A, Melen E, Vailes LD, Retief JD, Arruda LK, Chapman MD: Novel allergen structures with tandem amino acid repeats derived from German and American cockroach. J Biol Chem 1998, 273:30801-30807.

27. Wittstock U, Agerbirk N, Stauber EJ, Olsen CE, Hippler M, Mitchell-Olds T, Gershenzon J, Vogel H: Successful herbivore attack due to metabolic diversion of a plant chemical defense. Proc Natl Acad Sci USA 2004, 101:4859-4864.

28. Fischer HM, Wheat $\mathrm{CW}$, Heckel DG, Vogel H: Evolutionary origins of a novel host plant detoxification gene in butterflies. Mol Biol Evol 2008, 25:809-820.
29. Abraham EG, Islam S, Srinivasan P, Ghosh AK, Valenzuela JG, Ribeiro JM, Kafatos FC, Dimopoulos G, Jacobs-Lorena M: Analysis of the Plasmodium and Anopheles transcriptional repertoire during ookinete development and midgut invasion. J Biol Chem 2004, 279:5573-5580.

30. Shao L, Devenport M, Fujioka H, Ghosh A, Jacobs-Lorena M: Identification and characterization of a novel peritrophic matrix protein, Ae-Aper50, and the microvillar membrane protein, AEG12, from the mosquito, Aedes aegypti. Insect Biochem Molecul Biol 2005, 35:947-959.

31. Zaidman-Remy A, Herve M, Poidevin M, Pili-Floury S, Kim MS, Blanot D, Oh BD, Ueda R, Mengin-Lecreulx D, Lemaitre B: The Drosophila amidase PGRP-LB modulates the immune response to bacterial infection. Immunity 2006, 24:463-473.

32. Christophides GK, Zdobnov E, Barillas-Mury C, Birney E, Blandin S, Blass C, Brey PT, Collins FH, Danielli A, Dimopoulos G, Hetru C, Hoa NT, Hoffmann JA, Kanzok SM, Letunic I, Levashina EA, Loukeris TG, Lycett G, Meister S, Michel K, Moita LF, Müller HM, Osta MA, Paskewitz SM, Reichhart JM, Rzhetsky A, Troxler L, Vernick KD, Vlachou D, Volz J, von Mering C, Xu J, Zheng L, Bork P, Kafatos FC: Immunity-related genes and gene families in Anopheles gambiae. Science 2002, 298:159-165.

33. Meister S, Agianian B, Turlure F, Relogio A, Morlais I, Kafatos FC, Christophides GK: Anopheles gambiae PGRPLC-mediated defense against bacteria modulates infections with malaria parasites. PLOS Pathog 2009, 5: e1000542.

34. Warr E, Das S, Dong Y, Dimopoulos G: The Gram-negative bacteriabinding protein gene family: its role in the innate immune system of anopheles gambiae and in anti-Plasmodium defence. Insect Mol Biol 2008, 17:39-51.

35. Kumar S, Molina-Cruz A, Gupta L, Rodrigues J, Barillas-Mury C: A peroxidase/dual oxidase system modulates midgut epithelial immunity in Anopheles gambiae. Science 2010, 327:1644-1648.

36. Boulanger $N$, Lowenberger $C$, Volf $P$, Ursic R, Sigutova $L$, Sabatier $L$, Svobodova M, Beverley SM, Späth G, Brun R, Pesson B, Bulet P: Characterization of a defensin from the sand fly Phlebotomus duboscqi induced by challenge with bacteria or the protozoan parasite Leishmania major. Infect Immun 2004, 72:7140-7146.

37. Kumar S, Christophides GK, Cantera R, Charles B, Han YS, Meister S, Dimopoulos $\mathrm{G}$, Kafatos FC, Barillas-Mury C: The role of reactive oxygen species on Plasmodium melanotic encapsulation in Anopheles gambiae. Proc Natl Acad Sci USA 2003, 100:14139-14144

38. Zdobnov EM, von MC, Letunic I, Torrents D, Suyama M, Copley RR, Christophides GK, Thomasova D, Holt RA, Subramanian GM, Mueller HM, Dimopoulos G, Law JH, Wells MA, Birney E, Charlab R, Halpern AL, Kokoza E, Kraft CL, Lai Z, Lewis S, Louis C, Barillas-Mury C, Nusskern D, Rubin GM, Salzberg SL, Sutton GG, Topalis P, Wides R, Wincker P, Yandell M, Collins FH, Ribeiro J, Gelbart WM, Kafatos FC, Bork P: Comparative genome and proteome analysis of Anopheles gambiae and Drosophila melanogaster. Science 2002, 298:149-159.

39. Kanzok SM, Fechner A, Bauer H, Ulschmid JK, Muller HM, Botella-Munoz J, Schneuwly S, Schirmer R, Becker K: Substitution of the thioredoxin system for glutathione reductase in Drosophila melanogaster. Science 2001, 291:643-646.

40. Dillon RJ, Ivens AC, Churcher C, Holroyd N, Quail MA, Rogers ME, Soares MB, Bonaldo MF, Casavant TL, Lehane MJ, Bates PA: Analysis of ESTs from Lutzomyia longipalpis sand flies and their contribution toward understanding the insect-parasite relationship. Genomics 2006, 88:831-840.

41. Jaramillo-Gutierrez G, Rodrigues J, Ndikuyeze G, Povelones M, MolinaCruz A, Barillas-Mury C: Mosquito immune responses and compatibility between Plasmodium parasites and anopheline mosquitoes. BMC Microbiol 2009, 9:154

42. Narasimhan $S$, Sukumaran B, Bozdogan U, Thomas V, Liang X, Deponte $K$, Marcantonio N, Koski RA, Anderson JF, Kantor F, Fikrig E: A tick antioxidant facilitates the Lyme disease agent's successful migration from the mammalian host to the arthropod vector. Cell Host Microbe 2007, 2:7-18.

43. Molina-Cruz A, DeJong RJ, Charles B, Gupta L, Kumar S, JaramilloGutierrez G, Barillas-Mury C: Reactive oxygen species modulate Anopheles gambiae immunity against bacteria and Plasmodium. J Biol Chem 2008, 283:3217-3223.

44. Sant'Anna MR, Alexander B, Bates PA, Dillon RJ: Gene silencing in phlebotomine sand flies: Xanthine dehydrogenase knock down by dsRNA microinjections. Insect Biochem Mol Biol 2008, 38:652-660. 
45. Dunkov B, Georgieva T: Insect iron binding proteins: Insights from the genomes. Insect Biochem Mol Biol 2006, 36:300-309.

46. Ramalho-Ortigao JM, Traub-Cseko YM: Molecular characterization of LIchit1, a midgut chitinase CDNA from the leishmaniasis vector Lutzomyia longipalpis. Insect Biochem Mol Biol 2003, 33:279-287.

47. Sadlova J, Volf P: Peritrophic matrix of Phlebotomus duboscqi and its kinetics during Leishmania major development. Cell Tissue Res 2009, 337:313-325

48. Dinglasan RR, Devenport M, Florens L, Johnson JR, McHugh CA, DonnellyDoman M, Carucci DJ, Yates JR, Jacobs-Lorena M: The Anopheles gambiae adult midgut peritrophic matrix proteome. Insect Biochem Mol Biol 2009, 39:125-134.

49. Lehane MJ: Peritrophic matrix structure and function. Annu Rev Entomol 1997.

50. Devenport M, Alvarenga PH, Shao L, Fujioka H, Bianconi ML, Oliveira PL, Jacobs-Lorena M: Identification of the Aedes aegypti peritrophic matrix protein AelMUCl as a heme-binding protein. Biochemistry 2006, 45:9540-9549.

51. Anderson JM, Oliveira F, Kamhawi S, Mans BJ, Reynoso D, Seitz AE, Lawyer P, Garfield M, Pham M, Valenzuela JG: Comparative salivary gland transcriptomics of sandfly vectors of visceral leishmaniasis. BMC Genomics 2006, 7:52.

52. Volf $\mathrm{P}$, Volfova $\mathrm{V}$ : Establishment and maintenance of sand fly colonies. $J$ Vector Ecol 2011, 36.

53. Francischetti IM, Calvo E, Andersen JF, Pham VM, Favreau AJ, Barbian KD, Romero A, Valenzuela JG, Ribeiro JM: Insight into the Sialome of the Bed Bug, Cimex lectularius. J Proteome Res 2010, 9:3820-3831.

54. Huang X, Madan A: CAP3: A DNA sequence assembly program. Genome Res 1999, 9:868-877.

55. Ewing B, Hillier L, Wendl MC, Green P: Base-calling of automated sequencer traces using phred. I. Accuracy assessment. Genome Res 1998, 8:175-185.

56. Ewing B, Green P: Base-calling of automated sequencer traces using phred. II. Error probabilities. Genome Res 1998, 8:186-194.

57. Altschul SF, Madden TL, Schaffer AA, Zhang J, Zhang Z, Miller W, Lipman DJ: Gapped BLAST and PSI-BLAST: a new generation of protein database search programs. Nucleic Acids Res 1997, 25:3389-3402.

58. Ashburner M, Ball CA, Blake JA, Botstein D, Butler H, Cherry JM, Davis AP, Dolinski K, Dwight SS, Eppig JT, Harris MA, Hill DP, Issel-Tarver L, Kasarskis A, Lewis S, Matese JC, Richardson JE, Ringwald M, Rubin GM, Sherlock G: Gene ontology: tool for the unification of biology. The Gene Ontology Consortium. Nat Genet 2000, 25:25-29.

59. Tatusov RL, Fedorova ND, Jackson JD, Jacobs AR, Kiryutin B, Koonin EV, Krylov DM, Mazumder R, Mekhedov SL, Nikolskaya AN, Rao BS, Smirnov S, Sverdlov AV, Vasudevan S, Wolf YI, Yin JJ, Natale DA: The COG database: an updated version includes eukaryotes. BMC Bioinformatics 2003, 4:41, Epub; 2003 Sep 11.

60. Schultz J, Copley RR, Doerks T, Ponting CP, Bork P: SMART: a web-based tool for the study of genetically mobile domains. Nucleic Acids Res 2000, 28:231-234.

61. Bateman A, Birney E, Durbin R, Eddy SR, Howe KL, Sonnhammer EL: The Pfam protein families database. Nucleic Acids Res 2000, 28:263-266.

62. Bendtsen JD, Nielsen H, von HG, Brunak S: Improved prediction of signal peptides: SignalP 3.0. J Mol Biol 2004, 340:783-795.

63. Sonnhammer EL, von HG, Krogh A: A hidden Markov model for predicting transmembrane helices in protein sequences. Proc Int Conf Intell Syst Mol Biol 1998, 6:175-182.

64. Julenius K, Molgaard A, Gupta R, Brunak S: Prediction, conservation analysis, and structural characterization of mammalian mucin-type Oglycosylation sites. Glycobiology 2005, 15:153-164.

65. Larkin MA, Blackshields G, Brown NP, Chenna R, McGettigan PA, McWilliam H, Valentin F, Wallace IM, Wilm A, Lopez R, Thompson JD, Gibson TJ, Higgins DG: Clustal W and Clustal X version 2.0. Bioinformatics 2007, 23:2947-2948.

66. Abascal F, Zardoya R, Posada D: ProtTest: selection of best-fit models of protein evolution. Bioinformatics 2005, 21:2104-2105.

67. Schmidt HA, Strimmer K, Vingron M, von HA: TREE-PUZZLE: maximum likelihood phylogenetic analysis using quartets and parallel computing. Bioinformatics 2002, 18:502-504
68. Tamura K, Dudley J, Nei M, Kumar S: MEGA4: Molecular Evolutionary Genetics Analysis (MEGA) software version 4.0. Mol Biol Evol 2007, 24:1596-1599

doi:10.1186/1471-2164-12-223

Cite this article as: Dostálová et al:: The midgut transcriptome of Phlebotomus (Larroussius) perniciosus, a vector of Leishmania infantum: comparison of sugar fed and blood fed sand flies. BMC Genomics 2011 12:223.

\section{Submit your next manuscript to BioMed Central and take full advantage of:}

- Convenient online submission

- Thorough peer review

- No space constraints or color figure charges

- Immediate publication on acceptance

- Inclusion in PubMed, CAS, Scopus and Google Scholar

- Research which is freely available for redistribution

Submit your manuscript at www.biomedcentral.com/submit
Ciomed Central 